\title{
Digitalisierung - Umbruch oder Fortentwicklung im Recht des geistigen Eigentums
}

\section{Linda Kuschel}

Die Digitalisierung scheint auf den ersten Blick der Antagonist des Rechts des geistigen Eigentums. Während durch die Anerkennung von subjektiven Rechten an immateriellen Gütern etwas grundsätzlich nicht-exklusives normativ einer bestimmten Person exklusiv zugewiesen wird, ist die tatsächliche Ausschließbarkeit Dritter von eben diesen Gütern durch die Digitalisierung, die Aufwand und Kosten für die Reproduktion und Verbreitung drastisch gesenkt hat, beinah aufgehoben worden. Treffend stellt Wolfgang Hoffmann-Riem fest, das Recht des geistigen Eigentums werde durch die fortschreitende Digitalisierung (insbesondere das Internet) ,jetzt gehörig durchgeschüttelt“. ${ }^{1}$ Aber gab es auch einen „Umbruch“? Denkbar wäre ebenso, dass das Recht zwar durchgeschüttelt wurde, aber dennoch, wenn auch ein wenig derangiert, an seinem ursprünglichen Platz verweilt. Endgültig beurteilen lässt sich das erst, wenn das Beben vorüber ist; die von der Digitalisierung ausgelösten tektonischen Verschiebungen sind allerdings noch lange nicht vorüber. Um die Frage „Umbruch oder Fortentwicklung im Recht des geistigen Eigentums“ beantworten zu können, muss das Blickfeld des „jetzt“ in zwei Richtungen erweitert werden: in die Vergangenheit und in die Zukunft. Wo befand sich das Recht des geistigen Eigentums vor der Digitalisierung - was sind seine Ursprünge und Grundsätze? Und: Wie könnten die Technologien von morgen das Recht des geistigen Eigentums prägen; haben sie das Potential, zu einem Umbruch zu führen? 


\section{A. Das Gestern: Ursprung und Grundsätze des Rechts am geistigen Eigentum}

Für das Recht des geistigen Eigentums ${ }^{2}$ sind technische Entwicklungen kein Fremdkörper. ${ }^{3}$ Im Gegenteil: Sowohl Urheberrecht als auch Patentrecht verdanken ihre Existenz letztlich (auch) dem technischen Fortschritt. ${ }^{4}$ Durch die Erfindung des Buchdrucks im 15. Jahrhundert wurde die Vervielfältigung und Verbreitung von Schriftwerken wesentlich schneller und kostengünstiger möglich. ${ }^{5}$ Staatlich verliehene Privilegien, die einer Person die exklusive Erlaubnis gaben, in einem Hoheitsgebiet eine Druckpresse zu betreiben (Gewerbeprivileg) oder ein bestimmtes Werk zu drucken (Bücherprivileg), sicherten dabei in erster Linie nicht die Interessen der Autoren, sondern die Investitionen der Betreiber der Druckpresse, um diesen einen Anreiz zu bieten, sich in einem bestimmten Gebiet niederzulassen und die dortige Wirtschaft zu bereichern. ${ }^{6}$ Auch für Er-

2 Der Aufsatz beschäftigt sich mit dem Urheberrecht und dem Patentrecht als den „paradigmatischen“ (Götting, GRUR 2006, 353) Rechten des geistigen Eigentums. Der Schwerpunkt liegt dabei auf dem Urheberrecht als dem am stärksten von der Digitalisierung beeinflussten Immaterialgüterrecht. Zum (umstrittenen) Begriff des geistigen Eigentums vgl. nur Götting, GRUR 2006, 353; Jänich, Geistiges Eigentum - eine Komplementärerscheinung zum Sacheigentum?, 2002.

3 Vgl. Goldhammer, Geistiges Eigentum im Verfassungsstaat und darüber hinaus: öffentlich-rechtliche Skizzen aktueller Herausforderungen, in: Goldhammer/Grünberger/Klippel, Geistiges Eigentum im Verfassungsstaat, 2016, S. 1, 20, der das Rechtsgebiet als „technikaffin“ bezeichnet. Vgl. auch Schulte/Moufang, PatG $₫ 1$ Rn. 16: der technische Charakter ist dem Erfindungsbegriff „bereits immanent“.

4 Vgl. für das Urheberrecht Hilty, Sündenbock Urheberrecht?, in: Ohly/Klippel, Geistiges Eigentum und Gemeinfreiheit, 2007, S. 107; Ohly, Urheberrecht zwischen Innovationsstimulierung und -verhinderung, in: Eifert/Hoffmann-Riem, Geistiges Eigentum und Innovation, 2008, S. 279, 284; Schricker, Urheberrecht auf dem Weg zur Informationsgesellschaft, 1997, S. 5. Vgl. auch Peukert, Kritik der Ontologie des Immaterialgüterrechts, 2018, S. 74 ff., der neben dem technologischen Fortschritt auch den kulturellen Wandel von der „namenlosen Nachahmung zum genialen Werk" und die moderne Marktwirtschaft als Voraussetzungen der Entstehung des Immaterialgüterrechts sieht.

5 Siehe hierzu P. Goldstein, Copyright's Highway, 2019, S. 25 f.; McLuhan, The Gutenberg Galaxy: the making of typographic man, 1971, S. $237 \mathrm{ff}$. $\mathrm{Zu}$ früheren Schutzmechanismen für geistige Leistungen siehe etwa Jänich, Geistiges Eigentum - eine Komplementärerscheinung zum Sacheigentum?, 2002, S. 7 ff.; Schickert, Der Schutz literarischer Urheberschaft im Rom der klassischen Antike, 2005.

6 Zum Privilegienwesen siehe nur Heymann, Die zeitliche Begrenzung des Urheberrechts, Sitzungsberichte der Preußischen Akademie der Wissenschaften, 1927, XI, S. 65 ff.; Seifert, NJW 1992, 1270, 1273. Vgl. auch Ann, GRUR Int 2004, 597; Drei- 
findungen konnte ein Privileg erteilt werden, das ein zeitlich begrenztes Monopol zur gewerblichen Benutzung gewährte. ${ }^{7}$ Es stellte eine Belohnung für die Mühen und Kosten des Erfinders dar und sollte so als Anreiz zur Erfindertätigkeit fungieren. ${ }^{8}$ Vom modernen Patent- und Urheberrecht, die ihrem Inhaber ein subjektives Recht gewähren, war das sich durch hoheitliche Erteilung auszeichnende Privilegienwesen allerdings noch recht weit entfernt. ${ }^{9}$

Das erste Patentgesetz, das „Statute of Monopolies“, entstand 1624 in England. ${ }^{10}$ Es legte die Gewerbefreiheit als Grundsatz fest, in den Patente als Monopole nur ausnahmsweise und mit entsprechender Rechtfertigung eingreifen durften. ${ }^{11}$ Die Rechtfertigung für diese Ausnahme war der Nutzen von Erfindung und erfinderischer Tätigkeit für die Allgemeinheit. ${ }^{12}$

Ein Umdenken im theoretischen Fundament des Rechts am Geistigen Eigentum erfolgte im 17. und 18. Jahrhundert. Beeinflusst von der Philosophie der Aufklärung und von Naturrechtstheorien, allen voran der Labour Theory von John Locke, ${ }^{13}$ entstand auch im Recht des geistigen Ei-

er/Schulze/Dreier, UrhG Vor Rn. 10; Jänich, Geistiges Eigentum - eine Komplementärerscheinung zum Sacheigentum?, 2002, S. $20 \mathrm{ff.}$

7 Vgl. Kraßer/Ann/Ann, Patentrecht, $\mathbb{4}$ Rn. 12; Osterrieth, Patentrecht, 5. Aufl. 2015, Rn. 77; Poblmann GRUR 1960, 272.

8 Götting, Gewerblicher Rechtsschutz, 10. Aufl. 2014, S. 16 Rn. 13; Osterrieth, PatR, Rn. 78.

9 Vgl. Heymann, Die zeitliche Begrenzung des Urheberrechts, Sitzungsberichte der Preußischen Akademie der Wissenschaften, 1927, XI, 97 (im Kontext der urheberrechtlichen Schutzdauer); Runge GRUR 1948, 155; Seifert NJW 1992, 1270, 1273 ff.; Dölemeyer/Klippel, Der Beitrag der deutschen Rechtswissenschaft zur Theorie des gewerblichen Rechtsschutzes und Urheberrechts, in: Beier et al., FS GRUR Bd. I, 1991, S. 185, 189 ff. („prinzipieller Gegensatz“); vgl. aber auch Klippel, ZGE 1 (2015), 49, 55 f., der feststellt, dass es sich bei den Privilegien zumindest ihrer Wirkung nach um subjektive Rechte handelte, und Schack, Urheberund Urhebervertragsrecht, 9. Aufl. 2019, Rn. 110, der anmerkt, dass sich im Privilegienwesen bereits Andeutungen eines Urheberpersönlichkeitsschutzes finden.

10 Silberstein, Erfindungsschutz und merkantilistische Gewerbeprivilegien, 1961, S. $202 \mathrm{ff}$.

11 Kraßer/Ann/Ann, Patentrecht, $\mathbb{} 4$ Rn. 18 f.; Ann GRUR Int 2004, 597, 600.

12 Götting, Gewerblicher Rechtsschutz, S. 18 Rn. $18 \mathrm{f}$.

13 "Though the Earth, and all inferior Creatures be common to all Men, yet every Man has a Property in his own Person. This no Body has any Right to but himself. The Labour of his Body, and the Work of his Hands, we may say, are properly his. Whatsoever the he removes out of the State that Nature hath provided, and left in it, he hath mixed his Labour with it, and joined to it something that is his own, and thereby makes it his Property.“, Locke, Two Treatises of Government, Second Treatise, V, Abschnitt 26, 1689. 
gentums die Idee von einem „natürlichen“ Recht des Erfinders bzw. Autors, der - zwar nicht mit seinen Händen, aber mit seinem Geist - etwas Neues erschafft. ${ }^{14}$ Das erste Gesetz, das dem Autor und nicht mehr allein dem Verleger ein Recht an seinem Werk zugestand, war das britische Statute of Anne von $1710 .{ }^{15}$ In Frankreich vollzog sich der Wandel im Zuge der Revolution, die sämtliche Privilegien des Ancien régimes abschaffte. ${ }^{16}$ $1791^{17}$ und $1793^{18}$ werden Gesetze zum Schutz der Rechte am Propriété littéraire et artistique erlassen. ${ }^{19}$ Die von Le Chapelier in seinem Bericht von 1791 gebrauchte Formulierung, das Werk des Autors sei „la plus sacrée, la plus légitime, la plus inattaquable et [...] la plus personnelle de toutes les propriétés“20 wird - obwohl Le Chapelier sehr wohl auch die Interessen der Allgemeinheit vor Augen hatte - zum prägenden Motiv des kontinentaleuropäischen Urheberrechts. ${ }^{21}$ Parallel dazu rückte in der Epoche der Romantik das Individuum stärker in den Vordergrund und der sich in Musik, Literatur und bildender Kunst herauskristallisierende Geniegedanke beeinflusste vor allem das Urheberrecht maßgeblich. ${ }^{22}$ In Deutschland entwickelte sich das Recht am geistigen Eigentum aufgrund der Zersplitterung

14 Vgl. Dölemeyer/Klippel, Der Beitrag der deutschen Rechtswissenschaft zur Theorie des gewerblichen Rechtsschutzes und Urheberrechts, in: Beier et al., FS GRUR Bd. I, 1991, S. 185, 198 ff.; Kirchhof, Der verfassungsrechtliche Gehalt des geistigen Eigentums, in: Fürst et al., FS Zeidler, 1987, Bd. 2, S. 1639, 1640; Wadle, Der Weg zum gesetzlichen Schutz des geistigen und gewerblichen Schaffens, in: Beier et al., FS GRUR Bd. I, S. 98.

15 An Act for the Encouragement of Learning, by Vesting the Copies of Printed Books in the Authors or Purchasers of such Copies, during the Times therein mentioned. 8 Ann. c. 21.

16 Vgl. Osterrieth, PatR, Rn. 81; Ann, GRUR Int 2004, 597, $600 \mathrm{f}$.

17 Lois du 13 janvier 1791 et 19 juillet 1791, relative aux théâtres et au droit de représentation et d'exécution des ouvres dramatiques et musicales.

18 Décret de la Convention Nationale du dix-neuf juillet 1793 relatif aux droits de propriété des Auteurs d'écrits en tout genre, des Compositeurs de musique, des Peintres et des Dessinateurs.

19 Zur Bedeutung dieser Gesetze für die Vorstellung vom abstrakten Immaterialgut siehe Peukert, Kritik der Ontologie des Immaterialgüterrechts, 2018, S. 105 ff.

20 Le Chapelier's report, 1791, S. 16, Bentley/Kretschmer, Primary Sources on Copyright (1450-1900), abrufbar unter: http:/www.copyrighthistory.org/cam/tools/ request/showRecord?id=record_f_1791.

21 Vgl. Ginsburg, A Tale of Two Copyrights: Literary Property in Revolutionary France and America, 64 Tul. L. Rev. 991, 1006 ff. (1990).

22 Vgl. Bappert, Wege zum Urheberrecht, 1962, S. 105 ff.; Metzger, Vom Einzelurheber zu Teams und Netzwerken, in: Leible/Ohly/Zech, Wissen - Märkte - Geistiges Eigentum, 2010, S. 79, 80 f.; Peukert, Kritik der Ontologie des Immaterialgüterrechts, 2018, S. 80 ff.; Woodmansee, The Genius and the Copyright. Economic 
des Reiches nur langsam fort. ${ }^{23}$ Letztlich bildeten sich für das Urheberrecht einerseits und die gewerblichen Schutzrechte andererseits unterschiedliche Schwerpunkte in der Begründung heraus: ${ }^{24}$

Im Urheberrecht setzt sich die maßgeblich von Otto von Gierke ${ }^{25}$ geprägte Lehre vom Persönlichkeitsrecht durch. ${ }^{26}$ Sie wird im Rahmen der monistischen Theorie fortentwickelt, die das Urheberrecht als einheitliches Recht begreift, bestehend aus vermögensrechtlichen und persönlichkeitsrechtlichen Bestandteilen. ${ }^{27}$ Dieses theoretische Fundament spiegelt sich in den wichtigsten Regelungen und Grundsätzen des heutigen Urheberrechts wider: Urheberrechtsschutz wird nur für Werke als persönliche geistige Schöpfungen gewährt ( $\$ 2$ Abs. 2 UrhG), also insbesondere nur für von natürlichen Personen geschaffene Gegenstände. Der Urheberrechtsschutz bezieht sich auf die Form und den Ausdruck, nicht auf die dahinterliegende Idee. ${ }^{28}$ Das Urheberrecht entsteht formlos unmittelbar durch den Schöpfungsakt, ohne dass es etwa einer Registrierung bedürfte. ${ }^{29}$ Der Urheber wird sowohl in seinen persönlichen und geistigen Beziehungen zum Werk als auch in der wirtschaftlichen Nutzung des Werkes geschützt. Diese beiden Bestandteile des Urheberrechtsschutzes (Urheberpersönlichkeitsrechte und Verwertungsrechte) sind, entsprechend der monistischen Theorie, untrennbar miteinander verbunden. Daraus folgt, dass das Urheberrecht insgesamt unübertragbar ist ( $\mathbb{2} 29$ Abs. 1 UrhG). Auch die Schutzdauer des Urheberrechts orientiert sich an der Person des Schöpfers und gewährt noch 70 Jahre über den Tod des Autors hinaus Schutz ( $\$ 64$ UrhG).

Im Patentrecht steht von Beginn an der Gedanke des Nutzens für die Allgemeinheit stärker im Vordergrund. Die gewerblichen Schutzrechte sind als staatlich verliehene Monopole auch daran zu messen, ob der volks-

and Legal Conditions of the Emergence of the 'Author', Eighteenth-Century Studies, 17 (1984), 424.

23 Vgl. Götting, Gewerblicher Rechtsschutz, S. 23 f. Rn. 34.

24 Vgl. zum Urheberrecht Schricker/Loewenheim/Vogel, UrhG Einl. Rn. 127 ff., zu den gewerblichen Schutzrechten Götting, Gewerblicher Rechtsschutz, S. 25 Rn. 39 ff.. Kritisch Schricker, GRUR 1992, 242, 245.

25 Siehe nur $v$. Gierke, Deutsches Privatrecht Bd. I, 1895, $\mathbb{8} 85$, S. 748 ff., 764 ff., abgedruckt in UFITA 125 (1994), 103.

26 Vgl. Schack, UrhR, Rn. 120; Vogel, GRUR 1994, 587, 588.

27 Vgl. Schack, UrhR, Rn. 120; Schricker/Loewenheim/Vogel, UrhG Einl. Rn. 130.

28 Dreier/Schulze/Schulze, UrhG $\$ 2$ Rn. 37; Schricker/Loewenheim/Loewenheim/ Leistner, UrhG $\$ 2$ Rn. 73.

29 Schricker/Loewenheim/Loewenheim/Peifer, UrhG $\$ 7$ Rn. 5; Wandtke/Bullinger/ Thum, UrhG, $\mathbb{\$} 7$ Rn. 5 f. 
wirtschaftliche Nutzen die Nachteile überwiegt. ${ }^{30}$ Dementsprechend sind im Patentrecht, neben der naturrechtlichen Eigentumstheorie, nach der zuvörderst dem Erfinder das Recht an seiner geistigen Leistung zusteht, vor allem die Anreiztheorie und die Offenbarungstheorie von großer Bedeutung: ${ }^{31}$ Die Gewährung eines ausschließlichen Rechts soll den Erfinder motivieren, geistig tätig zu werden und, im Rahmen der Patentanmeldung, seine Erfindung der Gesellschaft zu offenbaren. ${ }^{32}$ Dementsprechend gewährt das Patentrecht Schutz für Erfindungen auf dem Gebiet der Technik ( $\$ 1$ Abs. 1 PatG) nur, wenn sie neu $(\$ 3$ PatG) und für einen Fachmann nicht selbstverständlich sind, also eine gewisse „Erfindungshöhe“33 aufweisen ( $\$ 4$ PatG). Es gilt das Erfinderprinzip, ${ }^{34}$ wonach die Person, die durch ihre individuelle geistige Leistung die technische Erfindung gemacht hat, Inhaber des Erfinderrechts ist und Anspruch auf das Patent hat ( $\$ 6$ PatG). Die Erfindung ist bei der Anmeldung zu offenbaren ( $\$ 34$ Abs. 4 PatG) und wird nach 18 Monaten auch der Öffentlichkeit offengelegt ( $\$ 31$ Abs. 2 S. 1 Nr. 2 PatG).

\section{B. Das Heute: Eine Bestandsaufnahme der Veränderungen durch die Digitalisierung}

Die Digitalisierung hat die Anzahl potentieller Schutzgegenstände erweitert, neue Arten der (rechtmäßigen und unrechtmäßigen) Nutzung ermöglicht und die Art und Weise der Verfolgung von Schutzrechtsverletzungen verändert. Aber inwieweit ist hiermit auch eine qualitative Veränderung

30 Ensthaler, Gewerblicher Rechtsschutz und Urheberrecht, 3. Aufl. 2009, S. 115; Klippel, Die Idee des geistigen Eigentums, in: Wadle, Historische Studien zum Urheberrecht in Europa, 1993, S. 121, 137.

$31 \mathrm{Zu}$ den verschiedenen Patenttheorien siehe nur Kraßer/Ann/Ann, Patentrecht, $\mathbb{} 3$ Rn. 7 ff.

32 Vgl. Benkard/Rogge/Melullis, PatG Einl Rn. 1. Vgl. auch Hoffmann-Riem, Innovation und Recht, Recht und Innovation, 2016, S. 437 („Das Recht am geistigen Eigentum soll in dieser Sichtweise einen Anreiz zu innovativem Verhalten schaffen und einen Anreiz zur Geheimhaltung nehmen.“).

33 Benkard/Schäfers/Schwarz PatG $₫ 59$ Rn. 76; Mes/Mes, PatG $₫ 4$ Rn. 57 ff. Vgl. auch Benkard/Assendorf/Schmidt, PatG $\$ 4$ Rn. 17.

$34 \mathrm{Im}$ ersten deutschen Patentgesetz von 1877 galt hingegen noch das Anmelderprinzip ( $(3$ Patentgesetz 1877), was sich erst mit der Patentrechts-Reform 1936 änderte. Vgl. hierzu Schade, GRUR 1977, 390. 
des Rechts am geistigen Eigentum einhergegangen? Diese Frage soll die folgende überblicksartige Bestandsaufnahme ${ }^{35}$ klären.

\section{Schutzgegenstand}

Weder Urheberrecht noch Patentrecht beschränken sich auf analoge Schutzgegenstände. Ein digitales Werk - etwa ein E-Book oder ein digitaler Film - ist in gleicher Weise urheberrechtlich geschützt wie eine auf einem materiellen Träger erzeugte Schöpfung. ${ }^{36}$ Eine Erfindung auf dem Gebiet der Technik wiederum muss nicht rein mechanisch wirken, sondern kann sich auch elektrischer Energie, digitaler Funktionen oder des Internets bedienen. ${ }^{37}$

Ein originär digitaler Schutzgegenstand, der sowohl Urheberrecht als auch Patentrecht herausfordert, ist das Computerprogramm. Im Patentrecht werden weder mathematische Methoden noch Programme für Datenverarbeitungsanlagen als Erfindung angesehen ( $\mathbb{1}$ Abs. 3 Nr. 1 und 3 PatG). Dieser Ausschluss gilt jedoch nur, soweit für diese Gegenstände „als solche" Schutz begehrt wird (vgl. $\$ 1$ Abs. 4 PatG). Damit sind zwar Computerprogramme für sich nicht patentierbar - Computerprogramme, die als Mittel zur Lösung eines konkreten technischen Problems eingesetzt werden, sog. computerimplementierte Erfindungen, hingegen schon. ${ }^{38}$ Entscheidende Bedeutung kommt also der Frage zu, ob das Computerprogramm einen technischen Charakter hat; ${ }^{39}$ die genauen Anforderungen an die technischen Merkmale des Computerprogramms sind allerdings unklar. ${ }^{40}$

35 Siehe hierzu auch Becker/Dreier, Urheberrecht und digitale Technologie, 1994 (im Kontext der digitalen Verwertung); Depenheuer/Peifer, Geistiges Eigentum: Schutzrecht oder Ausbeutungstitel?, 2008; Dreier/Leistner, GRUR-Beilage 2014, 13; Schricker, Urheberrecht auf dem Weg zur Informationsgesellschaft, 1997; Wandtke, UFITA 2011/III, S. 649.

36 Vgl. Schricker/Loewenheim/Loewenheim/Leistner, UrhG $\$ 2$ Rn. 48.

37 Vgl. Benkard/Bacher, PatG $\mathbb{1} 1$ Rn. 46a; Haedicke/Zech, GRUR-Beilage 2014, 52; Pesch MMR 2019, 14; Vgl. auch Zech, GRUR 2017, 475, der von einer „Dematerialisierung des Patentrechts"spricht.

38 Vgl. Götting, Gewerblicher Rechtsschutz, S. 131 Rn. 21; Schulte/Moufang, PatG $\ 1$ Rn. 111; Pesch, MMR 2019, 14.

39 Vgl. Götting, Gewerblicher Rechtsschutz, S. 131 Rn. 21; Osterrieth, PatR, Rn. 371 ff.; Schulte/Moufang, PatG $\$ 1$ Rn. 111.

40 Vgl. Schwarz/Kruspig, Computerimplementierte Erfindungen - Patentschutz von Software?, 2011, Kap. 3.5.8; Witte/Auer-Reinsdorff/Baldus, $\mathbb{5}$ Rechtsschutz von 
Das Urheberrecht ist - entsprechend seiner historischen Wurzeln - auf die individuelle Gestaltung eines (analogen oder digitalen) Gegenstands, also dessen Formgebung gerichtet. ${ }^{41}$ Die in einem Gegenstand enthaltenen Ideen oder Methoden bleiben frei. ${ }^{42}$ Gerade sie sind es allerdings, die in einem Computerprogramm von größtem Interesse sind, die konkrete Darstellung hingegen ist eher nebensächlich. ${ }^{43}$ Zudem unterscheiden sich Computerprogramme von anderen Werkarten in ihrer Bestimmung. Während bei Literatur, Musik oder Filmen der rezeptive Werkgenuss im Vordergrund steht, sind Computerprogramme durch ihre Funktionalität gekennzeichnet. Den hieraus folgenden Bedenken ${ }^{44}$ zum Trotz und anstatt eines sui generis-Schutzes, ${ }^{45}$ sind Computerprogramme heute als Sprachwerke geschützt ( $\$ 2$ Abs. $1 \mathrm{Nr} .1 \mathrm{UrhG}$ ). In den $\$ \$ 69 \mathrm{aff}$. UrhG, die die Vorgaben der Computerprogramme-Richtlinie ${ }^{46}$ umsetzen, finden sich zwar einige Sonderregelungen, die für Computerprogramme insofern leges speciales sind. ${ }^{47}$ In seinen wesentlichen Grundzügen unterscheidet sich der Schutz für Computerprogramme aber nicht von dem anderer Werkarten: Entsprechend der Trennung zwischen Form und Idee ${ }^{48}$ erstreckt sich der Schutz für ein Computerprogramm nur auf die Ausdrucksform und nicht auf die dem Programm zugrunde liegenden Ideen und Grundsätze ( $\$ 69 \mathrm{a}$ Abs. 2 UrhG). Nach $\$ 69$ b UrhG gehen bei Computerprogrammen, die in Arbeits- oder Dienstverhältnissen geschaffen wurden, alle vermögensrechtlichen Befugnisse automatisch auf den Arbeitgeber bzw. Dienstherren

Computerprogrammen und digitalen Inhalten, in: Auer-Reinsdorff/Conrad, IT-RHdB, 3. Aufl. 2019, Rn. 112 f.

41 Vgl. Dreier/Schulze/Schulze, UrhG \2 Rn. 37; Schack, UrhR, Rn. 187; Schricker/ Loewenheim/Loewenheim/Leistner, UrhG $₫ 2$ Rn. 47; Wandtke/Bullinger/Bullinger, UrhG $\$ 2$ Rn. 33.

42 Vgl. Schack, UrhR, Rn. 194; Schricker/Loewenheim/Loewenheim/Leistner, UrhG \2 Rn. 73; Wandtke/Bullinger/Bullinger, UrhG $\$ 2$ Rn. 19.

43 Cohen Jehoram, GRUR Int 1991, 687, 692.

44 Vgl. International Literary and Artistic Association (ALAI), 53. Kongress (Athen, 23-29 Mai 1976), Resolutionen in 1977 Industrial Property and Copyright, 197, 198. Vgl. auch Ricketson, The Berne Convention for the protection of literary and artistic works 1886 - 1986, 1987, S. 897 ff.

45 WIPO, Model Provisions on the Protection of Computer Software, 1977, in 1977 Industrial Property and Copyright, 259.

46 RL 91/250/EWG.

47 Vgl. Berger/Wündisch/Frank/Schulz, Urhebervertragsrecht, 2. Aufl. 2015, \$22 Rn. 12; Wandtke/Bullinger/Grützmacher, UrhG \$69a Rn. 1; Schricker/Loewenheim/Spindler, UrhG Vor $\$ \$ S 69$ a ff. Rn. 4 und 7; Rebbinder/Peukert, Urheberrecht, 18. Aufl. 2018, Rn. 599.

48 S.o. Fn. 41, 42. 
über; dennoch gilt auch für Computerprogramme das Schöpferprinzip, so dass die Erstrechtsinhaberschaft beim Urheber liegt. ${ }^{49}$

Diese Ausweitung des Urheberrechts durch den Schutz für Computerprogramme ist im Übrigen keine der Digitalisierung geschuldete Ausnahmeerscheinung, sondern fügt sich durchaus in eine lange Tradition der Schutzgegenstandserweiterung ein. ${ }^{50} \mathrm{Zu}$ nennen sind hier zum einen Auflockerungen des Werkbegriffs durch die Aufnahme von Werken der angewandten Kunst in den Begriff der bildenden Kunst ${ }^{51}$ und die kontinuierliche Absenkung der erforderlichen Gestaltungshöhe, ${ }^{52}$ zum anderen die Schaffung neuer Leistungsschutzrechte parallel zu Fortschritten auf technischem Gebiet, etwa zugunsten von Tonträgerherstellern, ${ }^{53}$ Presseverlegern $^{54}$ und Datenbankherstellern. ${ }^{55}$

\section{Ausschließlichkeitsrechte und Schranken}

Aus der verfassungsrechtlichen Institutsgarantie folgt für das geistige Eigentum die Pflicht des Gesetzgebers, „den Schutz der durch geistige Leistung geschaffenen Werke angesichts neuer technologischer und wirtschaft-

49 Vgl. Dreier/Schulze/Dreier, UrhG $\$ 69 b$ Rn. 2; Schricker/Loewenheim/Spindler, UrhG $₫ 69$ b Rn. 1; Wandtke/Bullinger/Grützmacher, UrhG $\ 69$ b Rn. 1.

50 Vgl. hierzu Cohen Jehoram, GRUR Int 1991, 687, 688.

51 Die Theorie der l'unité de l'art geht auf Pouillet, Traité théorique et pratique des dessins et modèles de fabrique, 5. Aufl. 1911, S. 52 (zitiert nach Perot-Morel, Les principes de protection des dessins et modèles dans les pays du Marché Commun, 1968, S. 39), zurück. Vgl. auch Passa, Droit de la propriété industrielle, 2009, Rn. 672.

52 Vgl. Schack, Weniger Urheberrecht ist mehr, in: Bullinger et al., FS Wandtke, 2013, S. 9, 12; Dreier/Schulze/Schulze, UrhG $\$ 2$ Rn. 22; Schricker/Loewenheim/ Loewenheim/Leistner, UrhG $\$ 2$ Rn. 60. Diese geringen Anforderungen gelten seit Umsetzung der RL 91/250/EWG auch für Computerprogramme und seit der BGH-Entscheidung Geburtstagszug (BGH, Urt. v. 13.11.2013, Az. I ZR 143/12 = BGHZ 199, 52) auch für Werke der angewandten Kunst.

53 S $85 \mathrm{f}$. UrhG, geschaffen mit dem Gesetz über Urheberrecht und verwandte Schutzrechte vom 9. September 1965 (BGBl. I S. 1273).

54 S\ $87 \mathrm{fff}$. UrhG, eingefügt durch 8. Gesetz zur Änderung des Urheberrechtsgesetzes vom 7. Mai 2013 (BGBl. I S. 1161). Die DSM-Richtlinie (RL (EU) 2019/790) sieht nun in Art. 15 ebenfalls ein Schutzrecht für Presseverleger vor.

55 S $\$$ 87a ff. UrhG, eingefügt durch Art. 7 Informations- und Kommunikationsdienste-Gesetz vom 22. Juli 1997 (BGBl. I S. 1870) in Umsetzung von Art. 7 Datenbank-RL des Europäischen Parlaments und des Rates vom 11.3.1996 über den rechtlichen Schutz von Datenbanken (ABl. 1996 L 77, 20 ff.). 
licher Gegebenheiten durch geeignete Regelungen zugunsten des Urhebers oder Erfinders zu gewährleisten. "56 Dementsprechend fallen neue Nutzungsmöglichkeiten, insbesondere durch technische Entwicklungen, in der Regel unmittelbar dem Ausschließlichkeitsrecht des Urhebers bzw. Erfinders zu. ${ }^{57}$

Die Digitalisierung hat neue Nutzungsformen für urheberrechtlich geschützte Werke ermöglicht. Sie unterliegen dem Ausschließlichkeitsrecht des Urhebers, ohne dass es einer entsprechenden Normierung durch den Gesetzgeber bedurfte: ${ }^{58}$ Das Vervielfältigungsrecht umfasst digitale Kopien;59 zum Recht der öffentlichen Wiedergabe zählen Online-Übertragungen, und zwar auch bevor diese explizit als Recht der öffentlichen Zugänglichmachung in $\$ 19$ a UrhG aufgenommen wurden. ${ }^{60} \mathrm{Im}$ Patentrecht spielen digitale Nutzungen keine vergleichbar große Rolle. ${ }^{61}$ Dennoch erschöpfen sich auch hier die Benutzungsbefugnisse nicht allein in analogen Handlungen, sondern können auch durch digitale Benutzungen - etwa einem Anbieten der Erfindung im Internet oder die (gewerbliche) Herstel-

56 Badura, Privatnützigkeit und Sozialbindung des geistigen Eigentums, in: Ohly/ Klippel, Geistiges Eigentum und Gemeinfreiheit, 2007, S. 45, 55. So auch st. Rspr. des BVerfG, vgl. zum Urheberrecht BVerfGE 31, 229, 238 ff. („Zu den konstituierenden Merkmalen des Urheberrechts als Eigentum im Sinne der Verfassung gehört die grundsätzliche Zuordnung des vermögenswerten Ergebnisses der schöpferischen Leistung an den Urheber im Wege privatrechtlicher Normierung und seine Freiheit, in eigener Verantwortung darüber verfügen zu können."); BVerfGE 49, 382; zum Patentrecht BVerfGE 36, 281, $290 \mathrm{f}$.

57 BGHZ 17, 266, 287 ff. Vgl. hierzu auch Badura, Privatnützigkeit und Sozialbindung des geistigen Eigentums, in: Ohly/Klippel, Geistiges Eigentum und Gemeinfreiheit, 2007, S. 45, 51.

58 Vgl. Dreier/Schulze/Schulze, UrhG $₫ 15$ Rn. 10 ff.; Spindler/Schuster/Wiebe, UrhG $\$ 15$ Rn. 1 f.; Wandtke/Bullinger/Heerma, UrhG $\$ 15$ Rn. 15 ff. Die Subsumtion neuer digitaler Nutzungswege unter die bestehenden Verwertungsrechte kann allerdings mitunter zu willkürlichen Ergebnissen führen, wenn etwa die Anzeige eines Programms auf einem analogen Fernseher urheberrechtlich nicht relevant ist, die Anzeige auf einem digitalen Fernseher, aufgrund der erforderlichen Vervielfältigungen, hingegen schon. Vgl. hierzu auch Dreier/Leistner, GRUR-Beilage 2014, 13, $18 \mathrm{f}$.

59 Vgl. Schack, UrhR, Rn.417; Schricker/Loewenheim/Loewenheim, UrhG $\$ 16$ Rn. 16.

60 Dreier/Schulze/Dreier, UrhG $₫ 19 a$ Rn. 3; Spindler/Schuster/Wiebe, UrhG $\$ 19 a$ Rn. 1; Wandtke/Bullinger/Bullinger, UrhG \$19a Rn. 2.

61 Vgl. Haedicke/Zech, GRUR-Beilage 2014, 52. 
lung patentrechtlich geschützter Gegenstände mithilfe von 3D-Druckern ${ }^{62}$ - betroffen sein. ${ }^{63}$

Für den Bereich der Ausschließlichkeitsrechte hat die Digitalisierung also zu einer quantitativen Erweiterung (vor allem im Urheberrecht) geführt. Eine qualitative Veränderung des Rechts ging hiermit jedoch nicht einher, weil der Zufluss neuer Nutzungsarten bereits im verfassungsrechtlichen Fundament des Rechts am geistigen Eigentum angelegt ist.

Im Gegensatz zu den Ausschließlichkeitsrechten sind die immaterialgüterrechtlichen Schranken grundsätzlich enumerativ und abschließend. ${ }^{64}$ Sie beziehen sich nicht automatisch auch auf neue, digitale Nutzungsarten, was dazu führt, dass die Schranken mit den Verwertungsrechten oft nicht Schritt halten können - auch wenn sie richtigerweise nach ihrem Sinn und Zweck zeitgemäß ausgelegt werden. ${ }^{65}$ Handlungen, die der Förderung der geistigen und kulturellen Werte dienen ${ }^{66}$ und folglich zugleich Grundlage für die Schöpfung neuer Werke sind, werden damit häufig erst nach (langwierigen) gesetzgeberischen Reformen legalisiert. ${ }^{67} \mathrm{Zu}$ den aufgrund der Digitalisierung neugeschaffenen Schranken im Urheberrecht zählen etwa die Erlaubnis vorübergehender Vervielfältigungen $(\mathbb{\$} 44 \mathrm{a}$ UrhG) und die Text und Data Mining-Schranke ( $\$ 60 \mathrm{~d}$ UrhG) ${ }^{68}$ Beide Schranken setzen, obgleich technisch motiviert, einen vorbestehenden Interessenausgleich um:

Der reine Werkgenuss - das Lesen eines Buches, das Anhören eines Musikstücks - ist urheberrechtlich frei, unterfällt also nicht den urheberrechtlichen Ausschließlichkeitsrechten. ${ }^{69}$ Der digitale Werkgenuss geht allerdings zwangsläufig mit Vervielfältigungen einher, weil die digitale Datei (zumindest kurzzeitig) im Arbeitsspeicher des Wiedergabegerätes gespei-

62 Vgl. hierzu Blanke-Roeser, GRUR 2017, 467; Haedicke/Zech, GRUR-Beilage 2014, 52, 55 f.; J.B. Nordemann/Rüberg/Schaefer, NJW 2015, 1265.

63 Vgl. Götting, Gewerblicher Rechtsschutz, S. 201 Rn. 9.

64 Vgl. Fromm/Nordemann/Dustmann, UrhG Vor $\$ 44 a$ Rn. 4; Schack, UrhR, Rn. 533.

65 Vgl. BGH, GRUR 2002, 963 - Elektronischer Pressespiegel; Hilty, GRUR 2005, 819, 823 f.; Geiger, Die Schranken des Urheberrechts im Lichte der Grundrechte - Zur Rechtsnatur der Beschränkungen des Urheberrechts, in: Hilty/Peukert, Interessenausgleich im Urheberrecht, 2004, S. $152 \mathrm{f}$.

66 Vgl. BT-Drucks. IV/270, S. 63.

67 Vgl. Dreier/Leistner, GRUR 2013, 881, 889; dies. GRUR-Beilage 2014, 13, 21.

68 Daneben umfassen manche Schranken, parallel zu analogen Befugnissen, digitale Nutzungsarten, so etwa die $\$ \$ \$ 60 \mathrm{aff}$. , vgl. Dreier/Schulze/Dreier, UrhG $\$ 60 \mathrm{a}$ Rn. 1.

69 Vgl. Wandtke/Bullinger/Heerma, UrhG $\$ 15$ Rn. 10. 
chert wird. ${ }^{70}$ Eben diese, für sich genommen wirtschaftlich irrelevanten, kurzzeitigen Speicherungen stellt $\$ 44$ a UrhG frei. Die Schranke übersetzt folglich die Werkgenussfreiheit ins Digitale. ${ }^{71}$

Die Schranke für Text und Data Mining in $\$ 60 \mathrm{~d} \mathrm{UrhG}^{72}$ ermöglicht eine softwaregestützte Auswertung großer Datenmengen. ${ }^{73}$ Zwar ist die Auswertung als solche keine urheberrechtlich relevante Handlung. ${ }^{74} \mathrm{Da}$ die Werke zuvor aber in ein maschinenlesbares Format (Korpus) überführt werden müssen, gehen mit dem Text und Data Mining in der Regel auch Vervielfältigungen einher. ${ }^{75}$ Diese technisch erforderlichen, wirtschaftlich aber irrelevanten Vervielfältigungen werden durch $\$ 60 \mathrm{~d}$ UrhG freigestellt und dadurch der Einsatz automatisierter Systeme erlaubt, der gegenüber einer manuellen Auswertung durch Personen wesentlich zeit- und kosteneffizienter ist und bestimmte Forschungsvorhaben überhaupt erst ermöglicht. ${ }^{76}$ Dennoch stellt die Text und Data Mining-Schranke keine qualitativ neue Beschränkung des Urheberrechts dar. Denn eine wichtige inhaltliche Begrenzung des Urheberrechtsschutzes ist die Differenzierung zwischen (geschützter) Form und (nicht geschützter) Information. Allein letztere ist beim Text und Data Mining betroffen. ${ }^{77}$ Zudem steht die Nutzung von

70 Vgl. Marly, EuZW 2014, 616; Rehbinder/Peukert, UrhR, Rn. 490.

71 Vgl. Wagner, GRUR 2016, 874, 878 f. Da \44a UrhG allerdings zwischen rechtmäßigen und unrechtmäßigen Quellen differenziert, werden analoger und digitaler Werkgenuss im Ergebnis doch nicht vollkommen gleichbehandelt, vgl. hierzu eingehend Sucker, Der digitale Werkgenuss im Urheberrecht, 2014; Kuschel, Der Erwerb digitaler Werkexemplare zur privaten Nutzung, 2019, S. 32 f. Vgl. auch Schack, UrhR, Rn. 412, der die Werkgenussfreiheit lediglich als Reflex ansieht.

72 Auch in Art. 3 f. der DSM-RL (RL (EU) 2019/790 über Urheberrecht und verwandte Schutzrechte im digitalen Binnenmarkt) sehen die Einführung einer Text und Data Mining Schranke verpflichtend vor. Die hiernach von den Mitgliedstaaten einzuführende Schranke geht teilweise über die bereits existierende deutsche Regelung hinaus. Siehe hierzu Hauk/Pflüger, ZUM 2020, 383, 385; Raue, ZUM 2019, 684; ders., ZUM 2020, 172.

73 Vgl. Amtl.Begr. BT-Drucks. 18/12329, S. 3. Siehe hierzu auch de la Durantaye, Allgemeine Bildungs- und Wissenschaftsschranke, 2014, S. $7 \mathrm{ff}$.

74 Vgl. Raue, ZUM 2019, 684, 685; Dreier/Schulze/Dreier, UrhG \$60d Rn. 1, 4; Schricker/Loewenheim/Stieper, UrhG $\$ 60 \mathrm{~d}$ Rn.3; Dreyer/Kotthoff/Meckel/ Hentsch, UrhG $\$ 60 \mathrm{~d}$ Rn. 3.

75 Vgl. Raue, CR 2017, 656; Spindler, GRUR 2016, 1112, 1113; ders., ZGE 10 (2018), 273, 276.

76 Vgl. Spindler, GRUR 2016, 1112.

77 Vgl. de la Durantaye, Allgemeine Bildungs- und Wissenschaftsschranke, 2014, S. 239. 
Text und Data Mining für die Zwecke wissenschaftlicher Forschung auch nicht in Konkurrenz zur normalen Werkverwertung. ${ }^{78}$

Dort, wo es an (rechtzeitig) neugeschaffenen Schranken fehlt, behilft sich die Rechtsprechung mit anderen Konstruktionen. ${ }^{79}$ So etwa bei der Vervielfältigung und öffentlichen Zugänglichmachung von Vorschaubildern durch Suchmaschinen: Hat der Rechtsinhaber Bilder (ohne technische Schutzmaßnahmen) öffentlich zugänglich gemacht, stelle dies eine „schlichte Einwilligung" in die Nutzung dar, da damit gerechnet werden müsse, dass die Bilder von den automatisierten Systemen der Suchmaschinen aufgefunden und verwendet würden. ${ }^{80}$

\section{Durchsetzung}

Die Digitalisierung ermöglicht es, Informationen komprimiert zu speichern, schnell und verlustfrei zu kopieren und sie über das Internet potentiell weltweit zugänglich zu machen. Insbesondere im Urheberrecht revolutionierten Digitalisierung und Vernetzung daher hergebrachte Verwertungsstrukturen. ${ }^{81}$ Vervielfältigung und öffentliche Wiedergabe liegen nicht mehr allein in den Händen klassischer Distributoren, wie etwa Verlagen, Fernseh- und Rundfunkanstalten, sondern können auch durch Priva-

78 Vgl. auch de la Durantaye, Allgemeine Bildungs- und Wissenschaftsschranke, 2014, S. 240; Schack, ZUM 2016, 266, 269. Dementsprechend sieht die DSM-RL anders als die deutsche Regelung in $\$ 60 d$ UrhG - für die Text und Data Mining Schranke auch keinen Ausgleich für die Rechtsinhaber vor, vgl. ErwG 17 DSMRL.

79 Vgl. hierzu Dreier/Leistner, GRUR-Beilage 2014, 13, 22; Leistner/Stang, CR 2008, 499, 503 ff.; Senftleben, 1 JIPITEC (2010), 67, 72 f.

80 BGH, GRUR 2010, 628 - Vorschaubilder I, und GRUR 2012, 602 - Vorschaubilder II. Eingehend hierzu Tinnefeld, Die Einwilligung in urheberrechtliche Nutzungen im Internet, 2012.

81 Vgl. nur Dreier/Leistner, GRUR-Beilage 2014, 13; Ernst, Reaktionen des Gesetzgebers, in: Dreier/Hilty, Vom Magnettonband zu Social Media, FS 50 Jahre UrhG, 2015, S. 195; Holzmüller/Staats, Verwertungsgesellschaften und Digitalisierung, in: Dreier/Hilty, Vom Magnettonband zu Social Media, FS 50 Jahre UrhG, 2015, S. 207; Harhoff/Hilty, Urheberrecht und Innovation in digitalen Märkten, Studie im Auftrag des BMJV, 2016; Lemley, IP in a World Without Scarcity, 90 NYU L. Rev. 460, 468 ff. (2015); Ohly, Urheberrecht in der digitalen Welt - Brauchen wir neue Regelungen zum Urheberrecht und dessen Durchsetzung?, Gutachten Teil F, in DJT, 70. Deutscher Juristentag, 2014. Vgl. auch Nagel, Wiederkehrende Argumente in der urheberrechtlichen Debatte um neue Technologien, in: Specht/ Lauber-Rönsberg/Becker, Medienrecht im Medienumbruch, 2017, S. 111. 
te und von beliebigen Orten aus erfolgen. Dementsprechend ist die Werkverwertung für den Rechteinhaber schwieriger zu kontrollieren und sind Rechtsverletzungen mitunter nur eingeschränkt verfolgbar. Vor diesem Hintergrund hat die Digitalisierung zu zwei wichtigen Veränderungen bei der Rechtsdurchsetzung geführt: Dem Einsatz technischer Schutzmaßnahmen und einer deutlich gestiegenen Relevanz der Störerhaftung im Recht des geistigen Eigentums.

Technische Schutzmaßnahmen sind „Technologien, Vorrichtungen und Bestandteile, die im normalen Betrieb dazu bestimmt sind, geschützte Werke oder andere nach diesem Gesetz geschützte Schutzgegenstände betreffende Handlungen, die vom Rechtsinhaber nicht genehmigt sind, zu verhindern oder einzuschränken ". ${ }^{82}$ Die technische Wirksamkeit dieser "faktischen Zugangsschranken" 83 wird rechtlich verstärkt: Nach $\$ 95 \mathrm{a}$ Abs. 1 UrhG dürfen technische Schutzmaßnahmen nicht ohne Zustimmung des Rechtsinhabers umgangen werden; entsprechende Vorbereitungshandlungen sind nach $\$ 95$ a Abs. 3 UrhG verboten. Ein Verstoß kann sowohl zivilrechtliche als auch strafrechtliche Konsequenzen nach sich ziehen. Problematisch ist der Einsatz technischer Schutzmaßnahmen dann, wenn diese nicht nur unerlaubte Handlungen, sondern auch urheberrechtlich gar nicht relevante oder von einer urheberrechtlichen Schranke gedeckte Handlungen verunmöglichen. ${ }^{84}$ Zwar gewährt $\$ 95$ b UrhG dem zur Nutzung Berechtigten einen Anspruch zur Durchsetzung bestimmter Schrankenbestimmungen gegenüber dem Verwender technischer Schutzmaßnahmen. Allerdings wird hiermit zum einen noch keine Aussage zu Handlungen getroffen, die gar kein urheberrechtliches Ausschließlichkeitsrecht betreffen, ${ }^{85}$ zum anderen werden längst nicht alle Schranken vollständig umfasst. ${ }^{86}$ So ist etwa die Privatkopieschranke nach $\$ 53$ Abs. 1 UrhG genannt, der Anspruch jedoch auf analoge Kopien beschränkt. ${ }^{87}$ Die Vorschrift steht zwar in der Kritik, ${ }^{88}$ wird jedoch überwiegend als verfassungskonform eingeschätzt, weil aus der Sozialpflichtigkeit des Eigentums

82 Legaldefinition in $\$ 95$ a Abs. 2 S. 1 UrhG.

83 Wielsch, Zugangsregeln, 2008, S. 15.

84 Vgl. hierzu Hilty, Sündenbock Urheberrecht?, in: Ohly/Klippel, Geistiges Eigentum und Gemeinfreiheit, 2007, S. 107, 116f.; Rehbinder/Peukert, UrhR, Rn. $1004 \mathrm{ff}$.

85 Vgl. hierzu Dreier/Leistner, GRUR-Beilage 2014, 13, 20.

86 Vgl. Dreier/Schulze/Specht, UrhG \$95b Rn. 12; Dreier, ZUM 2002, 28, 36 ff.

87 Vgl. Enders, ZUM 2004, 593, 601; Lauber-Rönsberg, Urheberrecht und Privatgebrauch, 2011, S. 215; Stickelbrock, GRUR 2004, 736, 740.

88 Holznagel/Brüggemann, MMR 2003, 767; Köcher/Kaufmann, MMR 2005, 753; Rigamonti, GRUR Int. 2005, 1, 9 (\$95b Nr. 6a UrhG sei völkerrechtswidrig); Hohagen, 
kein Anspruch auf freien Zugang zu Werken folge und die Informationsfreiheitsrechte wiederum nicht vor Eingriffen durch Private schützten. ${ }^{89}$ Im Übrigen wird den urheberrechtlichen Schranken ganz überwiegend lediglich rechtfertigender bzw. begrenzender Charakter beigemessen und ein korrespondierender Anspruch des Nutzers abgelehnt. ${ }^{90}$

Auch in der Rechtsverfolgung hat die Digitalisierung die Dynamik verändert. Insbesondere Urheberrechtsverletzungen im Internet stellen Rechteinhaber aufgrund der dezentralen Struktur des Netzwerks und der (potentiellen) Anonymität der Handelnden vor eine große Herausforderung. ${ }^{91}$ Anstatt gegen eine Vielzahl von unmittelbar Handelnden vorzugehen, werden vor allem Vermittler - Host-Provider, Access-Provider, Suchmaschinen - mithilfe des Instruments der Störerhaftung in die Pflicht genommen. ${ }^{92}$ Diese sind in der Regel einfacher zu erreichen als die unmittelbar Handelnden und können Rechtsverletzungen relativ kostengünstig un-

Überlegungen zur Rechtsnatur der Kopierfreiheit, in: Obly et al., FS Schricker, 2005, S. 353, 366 („völlig unakzeptabel“); Schweikart, UFITA 2005/I, 7, 12 ff.; Ulbricht, CR 2004, 674; Wandtke/Bullinger/Wandtke/Ohst, UrhG \$95b Rn. $4 \mathrm{f}$.

89 Arlt, Digital Rights Management Systeme, 2006, S. 201; v. Diemar, GRUR 2002, 587, 592; Stickelbrock, GRUR 2004, 736, 741. Vgl. auch Obly, Gesetzliche Schranken oder individueller Vertrag?, in: Dreier/Hilty, Vom Magnettonband zu Social Media, FS 50 Jahre UrhG, 2015, S. 379, 384, der sogar die Abschaffung der Privatkopie generell für verfassungskonform hält.

90 Arlt, Digital Rights Management Systeme, 2006, S. 203 (im Zusammenhang mit der Frage, ob sich aus $₫ 53$ UrhG ein Recht zur Umgehung technischer Schutzmaßnahmen ergibt); Stieper, Rechtfertigung, Rechtsnatur und Disponibilität der Schranken des Urheberrechts, 2009, S. 168 f.; Zech, Vertragliche Dispositionen über Schranken des geistigen Eigentums, in: Leible/Ohly/Zech, Wissen - Märkte - Geistiges Eigentum, 2010, S. 187, $188 \mathrm{f}$.

91 Vgl. nur Hennemann, Urheberrechtsdurchsetzung und Internet, 2011, S. 103; Nietsch, Anonymität und die Durchsetzung urheberrechtlicher Ansprüche im Internet, 2013, S. $68 \mathrm{ff}$.

92 Vgl. Hennemann, Urheberrechtsdurchsetzung und Internet, 2011, S. $108 \mathrm{ff}$; $\mathrm{Ku}$ schel, Netzsperren im Privatrecht, in: Mittwoch et al., Netzwerke im Privatrecht, 2016, S. 261; Leistner, GRUR 2006, 801; Spindler, Rechtliche Verantwortlichkeit nach Maßgabe technischer Kontrollmöglichkeiten? Das Beispiel der Verantwortlichkeit von Internet-Providern, in: Eifert/Hoffmann-Riem, Innovation, Recht und öffentliche Kommunikation, 2011, S. 80 ff.; G. Wagner, GRUR 2020, 329, 333. Vgl. auch Eifert, Das Netzwerkdurchsetzungsgesetz und Plattformregulierung, in: Eifert/Gostomzyk, Netzwerkrecht, 2018, S. 9, 12 („zentrale Verantwortungsstruktur"). 
terbinden. ${ }^{93}$ Allerdings führt das primäre Vorgehen gegen Intermediäre dazu, dass die Interessen der Handelnden, also etwa derjenigen, die einen bestimmten Inhalt erstellt und zugänglich gemacht haben, nicht hinreichend vertreten werden. Denn der Vermittler hat meist kein eigenes Interesse an einem konkreten Inhalt. ${ }^{94}$ Aus seiner Perspektive ist es daher ökonomisch sinnvoll, im Zweifel lieber zu viel als zu wenig zu sperren, um einer Haftung zu entgehen. ${ }^{95}$ Dies kann dazu führen, dass auch Inhalte, die urheberrechtlich unbedenklich sind, der Öffentlichkeit entzogen werden. ${ }^{96}$

\section{Zwischenbilanz}

Obwohl die Digitalisierung zweifellos Auswirkungen auf das Recht des geistigen Eigentums - allen voran das Urheberrecht - hatte, zeigt eine Analyse der bisherigen rechtlichen Veränderungen doch, dass eine Disruption bislang ausgeblieben ist. ${ }^{97}$ Der Kreis der Schutzgegenstände hat sich erwei-

$93 \mathrm{Zu}$ Intermediären als cheapest cost avoider vgl. Leistner, ZUM 2012, 722, 723; Dreier/Leistner, GRUR-Beilage 2014, 13, 26; Ohly, ZUM 2015, 308; G. Wagner, GRUR 2020, 329, 337 f. Ähnlich auch das BVerfG im Rahmen der Haftung von Suchmaschinenbetreibern für Drittinhalte (dies sei „effizienter"), s. BVerfG GRUR 2020, 88 Rn. 119 - Recht auf Vergessen II.

94 Vgl. Magnus, Dürfen schlaue Trolle das ganze Internet kaputtmachen? - Der Anspruch auf Wiederherstellung von Nutzerbeiträgen, in: Hetmank/Rechenberg, Kommunikation, Kreation und Innovation - Recht im Umbruch?, 2019, S. 99, 100; Nolte, ZUM 2017, 552, 556 („kein nennenswerter wirtschaftlicher Wert“); Raue, JZ 2018, 961, 963. Allerdings haben viele Plattformen zumindest ein Interesse, möglichst viele Inhalte bereitzustellen, vgl. Gerpott, MMR 2019, 420, 422; Pravemann, GRUR 2019, 783, 788.

95 Vgl. F. Hofmann, GRUR 2019, 1219, 1227; D. Holznagel, Notice and Take-DownVerfahren als Teil der Providerhaftung, 2013, S. $206 \mathrm{ff}$;; Kuschel, Netzsperren im Privatrecht, in: Mittwoch et al., Netzwerke im Privatrecht, 2016, S. 261, 288 f.; Raue, JZ 2018, 961, 963. Differenzierend im Zusammenhang mit den Pflichten von Internetplattformen nach dem Netzwerkdurchsetzungsgesetz Eifert, Das Netzwerkdurchsetzungsgesetz und Plattformregulierung, in: Eifert/Gostomzyk, Netzwerkrecht, 2018, S. 9, 34 ff.; Lang, AöR 143 (2018), 220, 232 ff.

96 Vgl. Hoffmann-Riem, Kommunikationsfreiheiten, 2002, S. 145; Kastl, GRUR 2016, 671, 678; Leistner/Grisse, GRUR 2014, 105, 108 ff.; Mantz, GRUR 2017, 969; Nolte/ Wimmers, GRUR 2014, 16.

97 So auch Nagel, Wiederkehrende Argumente in der urheberrechtlichen Debatte um neue Technologien, in: Specht/Lauber-Rönsberg/Becker, Medienrecht im Medienumbruch, 2017, S. 111. Für das Marken- und Lauterkeitsrecht (in Bezug auf die Herausforderungen durch das Internet) Glöckner/Kur, GRUR-Beilage 2014, 29. 
tert, die Verwertungsrechte des Rechtsinhabers sind parallel zu neuen Nutzungsmöglichkeiten angewachsen, neugeschaffene Schrankenregelungen übersetzen Interessenabwägungen aus der analogen Welt ins Digitale und die Mittel zur Rechtsdurchsetzung folgen der gewandelten Realität der Nutzung und Verbreitung von Inhalten.

Die zu Anfang des 21. Jahrhunderts prophezeiten großen Revolutionen und anvisierten grundlegenden Reformen ${ }^{98}$ sind aber im Wesentlichen ausgeblieben. Insbesondere hat der Schutz des geistigen Eigentums keineswegs an Relevanz verloren oder sich "aufgelöst“.99 Vorschläge für neue, pauschale Verwertungsmodelle, etwa im Rahmen einer Kultur-Flatrate, ${ }^{100}$ wurden ebenso wenig umgesetzt wie ein Wechsel vom Katalog der Einzelschranken zu einer Generalklausel nach Vorbild des US-amerikanischen Fair Use-Systems. ${ }^{101}$ Die Open-Content-Bewegung hat zwar hergebrachte

98 Vgl. etwa Schlussbericht der Enquete-Kommission Internet und digitale Gesellschaft, April 2013, BT-Drucks. 17/12550. Hargreaves, Digital Opportunity: A Review of Intellectual Property and Growth, Mai 2011, abrufbar unter www.gov.uk/government/publications/digital-opportunity-review-of-intellectualproperty-and-growth. Auch auf europäischer und internationaler Ebene strebte man Reformen an: Die Vertragsstaaten des EPÜ verhandelten im Jahr 2000 über einen Basisvorschlag für die Revision des EPÜ (MR/2/00), der u.a. vorsah, die Ausnahme des Art. 52 Abs. 2 lit. c EPÜ („Programme für Datenverarbeitungsanlagen“) zu entfernen. Die EU plante mit dem Vorschlag für eine Richtlinie des Europäischen Parlaments und des Rates über die Patentierbarkeit computerimplementierter Erfindungen (KOM/2002/0092 endg.) von 2002, computerimplementierte Erfindungen zu schützen, wenn sie „einen technischen Beitrag zum Stand der Technik“ leisteten (Art. 4). Der Vorschlag scheiterte 2005 an der mangelnden Zustimmung des Parlaments.

99 Vgl. Geller, Dissolving Intellectual Property, 2006, abrufbar unter: https://pgeller .com/Paul_Geller-Dissolving_Intellectual_Property.pdf; Smiers/van Schijndel, Imagine there is no copyright and no cultural conglomerates too, 2009, abrufbar unter: https://networkcultures.org/_uploads/tod/TOD4_nocopyright.pdf.

100 BT-Drucks. 17/7899, S. 47 f.; Spindler, Rechtsprobleme und wirtschaftliche Vertretbarkeit einer Kulturflatrate, 2014, abrufbar unter: https://univerlag.uni-goetti ngen.de/bitstream/handle/3/isbn-978-3-86395-128-3/Spindler_Kulturflatrate.pdf?s equence=1\&. Vgl. hierzu auch Roßnagel/Jandt/Schnabel, MM $\bar{R}$ 2010, 8. Kritisch Schwartmann/Hentsch, ZUM 2012, 759, $768 \mathrm{f}$.

101 BT-Drucks. 17/7899, S. 21. Vgl. hierzu auch Förster, Fair Use, 2008, S. 214 f.; Hoeren, GRUR 1997, 866, 871; Metzger, Urheberrechtsschranken in der Wissensgesellschaft: „Fair use“ oder enge Einzeltatbestände?, in: Leistner, Europäische Perspektiven des Geistigen Eigentums, 2010, S. 101, 118 ff.; Senftleben, JIPITEC 1 (2010), S. 67 Rn. 43 ff. Kritisch Schwartmann/Hentsch, ZUM 2012, 759, 765 f. 
Begründungsansätze in Frage gestellt. ${ }^{102}$ Sie hat sich dabei aber das bestehende System zunutze gemacht und durch (lizenz-)vertragliche Regelungen an ihre Bedürfnisse angepasst; ${ }^{103}$ eine Veränderung des Rechts hat sie nicht bewirkt. ${ }^{104}$

Die bisherigen Veränderungen des Rechts am geistigen Eigentum waren also eher Fortentwicklung als Umbruch. Obgleich durchgeschüttelt, ist das Recht des geistigen Eigentums recht beharrlich an seinem Platz geblieben; es steht allerdings ordentlich windschief. Denn während sich etwa der Schutzgegenstand deutlich erweitert und - insbesondere im Urheberrecht - vom ursprünglichen Kern weit entfernt hat, ist dessen theoretische Begründung weitestgehend unverändert geblieben. Noch heute spiegelt sich in den Grundsätzen des Urheberrechts - Schöpferprinzip, Unübertragbarkeit, Schutzdauer - vor allem die Persönlichkeitstheorie wieder, ${ }^{105}$ auch wenn ökonomische Theorien zur Begründung des Rechts am geistigen Eigentum zunehmend an Boden gewinnen. ${ }^{106}$ Sowohl im Urheberrecht als auch im Patentrecht bildet die Fokussierung auf die Person des (Einzel-)Schöpfers bzw. des (Einzel-)Erfinders die Realitäten des Entstehungsprozesses von vielen Werken und Erfindungen nicht mehr hinreichend ab. ${ }^{107}$ Die lange Schutzdauer und umfassenden Ausschließlichkeitsrechte

102 Vgl. Bechtold, GRUR Int 2008, 484, 486; Hoffmann-Riem, Innovation und Recht, Recht und Innovation, 2016, S.441; Jaeger/Metzger, Open Source Software: Rechtliche Rahmenbedingungen der Freien Software, 2. Aufl. 2006, S. 82 ff.; Spindler, Open Source Software: Offene Rechtsfragen, in: Taeger/Wiebe, Informatik - Wirtschaft - Recht, Regulierung der Wissensgesellschaft, FS Kilian, 2004, S. $353 \mathrm{ff}$.

103 Vgl. Hoffmann-Riem, JZ 2012, 1081, 1085 („geniale Um-Erfindung“).

104 Vgl. hierzu Obly, Urheberrecht zwischen Innovationsstimulierung und -verhinderung, in: Eifert/Hoffmann-Riem, Geistiges Eigentum und Innovation, 2008, S. 279, 282.

105 Vgl. Lauber-Rönsberg, GRUR 2019, 244, 250. Kritisch Dornis, GRUR 2019, 1252, 1258 f.; Leistner/Hansen GRUR 2008, 479, 480 f. Siehe aus rechtsvergleichender Sicht Ginsburg, The Concept of Authorship in Comparative Copyright Law, Columbia Law School Public Law and Legal Theory Research Paper Group, No. 03-51; dies., A Tale of Two Copyrights: Literary Property in Revolutionary France and America, 64 Tul. L. Rev. 991 (1990).

106 Vgl. Bechtold, GRUR Int 2008, 484; Grünberger/Jansen, Perspektiven deutscher Privatrechtstheorie, in: Grünberger/Jansen, Privatrechtstheorie heute, 2017, S. 1, 32; Leistner/Hansen, GRUR 2008, 479, 481 ff.; Nazari-Khanachayi, Rechtfertigungsnarrative des Urheberrechts im Praxistest, S. 65 ff.; Peukert, Güterzuordnung als Rechtsprinzip?, 2008, S. 94 ff.; ders., Kritik der Ontologie des Immaterialgüterrechts, 2018, S. 153 f.; Wielsch, Zugangsregeln, 2008, S. $13 \mathrm{ff}$.

107 Vgl. Dreier/Leistner, GRUR-Beilage 2014, 13, 17; Dornis, GRUR 2019, 1252, 1254 („geradezu romantische Vorstellung kreativen Schaffens“); Hoffmann-Riem, JZ 
wiederum hemmen Kreativität und Innovation teilweise mehr als dass sie sie fördern. ${ }^{108} \mathrm{Im}$ Urheberrecht (und insbesondere bei den verwandten Schutzrechten) ${ }^{109}$ wird der Tatsache, dass Schöpfungen immer auch auf Vorbestehendem aufbauen und mit steigender Zahl von Werken der Raum für Neuschöpfungen immer schmaler wird, zu wenig Beachtung geschenkt, was durch die „Demokratisierung der Kreativmittel“110 im Zuge der Digitalisierung und die Zunahme an referentieller Kunst im Internet besonders deutlich wird. ${ }^{111}$

\section{Das Morgen: Umbruch durch automatisierte und autonome Systeme?}

Bisherige Entwicklungsstufen der Digitalisierung - insbesondere die Umwandlung von analogen Medien in digitale und die Vernetzung durch das

2012, 1081, 1084.; W. Fisher, Geistiges Eigentum - ein ausufernder Rechtsbereich, in: Siegrist/Sugarman, Eigentum im internationalen Vergleich, 2011, S. 265, 281; Metzger, Vom Einzelurheber zu Teams und Netzwerken, in: Leible/Ohly/Zech, Wissen - Märkte - Geistiges Eigentum, 2010, S. 79, 88, der von einem tatsächlichen „Veränderungsdruck auf das Schöpferprinzip“ spricht; Peukert, Der digitale Urheber, in: Bullinger et al., FS Wandtke, 2013, S. 459.

108 Vgl. Hargreaves/Hugenholtz, Copyright Reform for Growth and Jobs: Modernising the European Copyright Framework, Interactive Policy Brief 13/2013, S. 8 f.; Hilty, Sündenbock Urheberrecht?, in: Ohly/Klippel, Geistiges Eigentum und Gemeinfreiheit, 2007, S. 107, 130 ff.; Hilty/Senftleben, Rückschnitt durch Differenzierung? - Wege zur Reduktion dysfunktionaler Effekte des Urheberrechts auf Kreativ- und Angebotsmärkte, in: Dreier/Hilty, Vom Magnettonband zu Social Media, FS 50 Jahre UrhG, 2015, S. 317, 318; Hoffmann-Riem, Innovation und Recht, Recht und Innovation, 2016, S. 433; Obly, Urheberrecht zwischen Innovationsstimulierung und -verhinderung, in: Eifert/Hoffmann-Riem, Geistiges Eigentum und Innovation, 2008, S. 279.

109 Nach Rechtsprechung des BGH ist etwa der Schutz von Laufbild- und Tonträgerherstellern bereits bei der Übernahme kleinster Bildausschnitte bzw. „Tonfetzen“ betroffen, vgl. BGH, GRUR 2008, 693 - TV Total; BGH, GRUR 2009, 403 Rn. 10 - Metall auf Metall I; BGH, GRUR 2017, 895 Rn. 16 - Metall auf Metall III; BGH, Urt. v. 30.04.2020, Az.: I ZR 115/16 - Metall auf Metall IV (noch nicht veröffentlicht). Vgl. hierzu Dreier/Leistner, GRUR-Beilage 2014, 13, 15.

110 Dreier/Leistner, GRUR-Beilage 2014, 13, 14.

111 Vgl. Dreier/Leistner, GRUR-Beilage 2014, 13, 14; Hoffmann-Riem, JZ 2012, 1081, 1084; ders., Innovation und Recht, Recht und Innovation, 2016, S. 441; Klass, ZUM 2016, 801; Lessig, Remix: Making Art and Commerce Thrive in the Hybrid Economy, 2008, S. 253 ff.; H. Maier, Remixe auf Hosting-Plattformen, 2018, S. 1; dies., GRUR-Prax 2016, 397; Papastefanou, WRP 2019, 171; Pötzlberger, Kreatives Remixing: Musik im Spannungsfeld von Urheberrecht und Kunstfreiheit, 2018, S. 26, 38 . 
Internet - haben das Recht des geistigen Eigentums zwar punktuell verändert, für wirklich große Umbrüche haben sie aber wohl ihr Momentum verloren. Damit stellt sich die Frage, ob Technologien von morgen (bzw. solche, die schon heute existieren, aber wahrscheinlich noch nicht ihr volles Potential entfaltet haben) zu einer Disruption im Recht des geistigen Eigentums führen könnten oder jedenfalls dazu Anlass bieten, alte Strukturen $\mathrm{zu}$ überdenken und gegebenenfalls Korrekturen vorzunehmen. ${ }^{112}$ Hierzu gehört insbesondere der Einsatz Künstlicher Intelligenz, ${ }^{113}$ also von Systemen, „die ihre Umgebung analysieren und mit einem gewissen Grad an Autonomie handeln, um bestimmte Ziele zu erreichen." ${ }^{114} \mathrm{KI}$ ist für das Recht des geistigen Eigentums in verschiedener Hinsicht eine Herausforderung: Zum einen, weil für die Systeme selbst ein immaterialgüterrechtlicher Schutz in Betracht kommt. ${ }^{115}$ Zum anderen, weil die von KI generierten Ergebnisse potentielle Schutzgegenstände sind. Daneben können autonome und automatisierte Systeme zur Rechtsverfolgung herangezogen werden ${ }^{116}$ und kommen, insbesondere in Form von Filtertechnologien, auch im Recht des geistigen Eigentums zum Einsatz.

112 Die im Folgenden angestellten Überlegungen sollen lediglich Denkanstöße sein, die im Rahmen der derzeit geltenden internationalen und europarechtlichen Vorgaben nicht unbedingt umsetzbar wären. In den Worten von Dreier/Leistner: „Denn Bindungen internationalen Rechts dürften wirklich innovativen Plänen beinahe stets entgegenstehen." (GRUR-Beilage 2014, 13, 21).

$113 \mathrm{Zu}$ Recht kritisch gegenüber der Weite des Begriffs der „Künstlichen Intelligenz" Gesmann-Nuissl, InTeR 2018, 105; Hauck/Cevc, ZGE 11 (2019), 135, 138 ff.; Herberger, NJW 2018, 2825.

114 Mitteilung der Kommission an das Europäische Parlament, den Rat, den Europäischen Wirtschafts- und Sozialausschuss und den Ausschuss der Regionen „Künstliche Intelligenz für Europa“, Brüssel, 25.4.2018, COM(2018) 237 (final), abrufbar unter: https://ec.europa.eu/transparency/regdoc/rep/1/2018/DE/COM-2 018-237-F1-DE-MAIN-PART-1.PDF. Für eine erweiterte und detailreichere Definition siehe Unabhängige Hochrangige Expertengruppe für Künstliche Intelligenz, Eine Definition der KI: Wichtigste Fähigkeiten und Wissenschaftsgebiete, April 2019, abrufbar unter: https://ec.europa.eu/newsroom/dae/document.cfm?d oc_id=60664. Eine ausführliche Begriffsbestimmung nimmt auch Linke, Urheberrechtlicher Schutz von „KI“ als Computerprogramme - Squeezing today’s innovations into yesterday's system?, in: Hetmank/Rechenberg, Kommunikation, Kreation und Innovation - Recht im Umbruch?, 2019, S. 29, 31 ff. vor.

115 Daneben kommt auch ein Schutz als Geschäftsgeheimnis in Betracht, vgl. hierzu Scheja, CR 2018, 485.

116 Zur automatisierten Rechtsdurchsetzung im Privatrecht siehe etwa Fries, NJW 2016, 2860, 2861 ff.; Kuschel, AcP 220 (2020), 98; Specht, GRUR 2019, 253, 255; Stiemerling, CR 2015, 762. 


\section{Systeme Künstlicher Intelligenz}

Die Fähigkeiten von KI beruhen auf der Zusammenführung verschiedener Komponenten: leistungsfähiger Hardware, Software, die auf mathematischen Modellen und Algorithmen basiert, und großer Mengen an Daten bzw. Datensätzen, mit denen die Software trainiert wird. ${ }^{117}$

Naheliegend erscheint ein Schutz für KI durch das Patentrecht. ${ }^{118}$ Allerdings werden nach $₫ 1$ Abs. 3 Nr. 1 PatG (und Art. 52(2)(a) EPÜ) mathematische Methoden nicht als Erfindungen angesehen. Dementsprechend sind auch die KI-Systemen zugrundeliegenden mathematischen Modelle und Algorithmen nicht als solche patentfähig. ${ }^{119}$ Die konkrete Anwendung von Algorithmen und mathematischen Modellen in einem KI-System kann aber durchaus technischen Gehalt haben und dementsprechend schutzfähig sein. Die Diskussion um die Schutzfähigkeit von KI-Systemen läuft letztlich, wie bei Computerprogrammen, die nur als computerimplementierte Erfindungen schutzfähig sind, ${ }^{120}$ auf die Frage hinaus, ob das System ein technisches Problem mit technischen Mitteln löst. ${ }^{121}$ Entscheidende Stellschraube ist dabei der Begriff der Technizität. Hier hat in den vergangenen Jahrzehnten bereits eine deutliche Auflockerung des ursprünglich recht strengen Technikverständnisses stattgefunden. ${ }^{122}$ Durch

117 Vgl. hierzu Fink, ZGE 9 (2017), 288, 289 ff.; Hauck/Cevc, ZGE 11 (2019), 135, 144.

118 Siehe hierzu Abbott, I Think, Therefore I Invent: Creative Computers and the Future of Patent Law, 57 B. C. L. Rev. 1079 (2016); Clifford, Intellectual Property in the Era of the Creative Computer Program, 71 Tul. L. Rev. 1675, 1695 (1997); Hauck/Cevc, ZGE 11 (2019), 135; Hetmank/Lauber-Rönsberg, GRUR 2018, 574, 575; Lederer, GRUR-Prax 2019, 152; Ménière/Pihlajamaa, GRUR 2019, 332; Nägerl/Neuburger/Steinbach, GRUR 2019, 336; Papastefanou, „Machine Learning“ im Patentrecht - Herausforderungen beim Erfinderbegriff und der Patentierfähigkeit von Algorithmen, in: Hetmank/Rechenberg, Kommunikation, Kreation und Innovation - Recht im Umbruch?, 2019, S. 9; Zech/Lauber-Rönsberg/ Hetmank, GRUR Newsletter 02/2017, 17.

119 Vgl. Hauck/Cevc, ZGE 11 (2019), 135, 147; Ménière/Pihlajamaa, GRUR 2019, 332, 334; Nägerl/Neuburger/Steinbach, GRUR 2019, 336, 339; Zech, Technizität im Patentrecht - Eine intra- und interdisziplinäre Analyse des Technikbegriffs, Metzger, Methodenfragen des Patentrechts, FS Bodewig, 2018, S. 137, 151. Vgl. auch EPA, Richtlinien für die Prüfung im Europäischen Patentamt, November 2019, Teil G, Kap. II, 3.3.

120 So auch Hauck/Cevc, ZGE 11 (2019), 135, 148.

121 BGHZ 117, 144 - Tauchcomputer; BGH, GRUR 2010, 613 - Dynamische Dokumentengenerierung; BGH, GRUR 2011, 610 - Webseitenanzeige.

122 Vgl. BeckOK-PatR/Einsele, PatG $\$ 1$ Rn. 20 ff.; Benkard/Asendorf/Schmidt, PatG \ 4 Rn. 59 ff.; Benkard/Melullis, EPÜ Art. 52 Rn. 275; Pesch, MMR 2019, 14, 15 f. 
die stetig weiter zunehmende Relevanz digitaler, also unkörperlicher Gegenstände, ist perspektivisch eine noch stärkere „Bedeutungsverschiebung des Technikbegriffs" 123 denkbar. Daneben könnte die Thematik der Schutzfähigkeit von KI eine Gelegenheit bieten, das formale Technizitätserfordernis generell zu hinterfragen ${ }^{124}$ und sich stärker auf die mit dem Patentschutz verfolgten Ziele - insbesondere die Innovationsförderung - zu konzentrieren. Neben dem Innovationsanreiz hätte die Patentierung von KI-Systemen im Übrigen noch einen weiteren wichtigen Vorteil: Entwicklern solcher Systeme würde ein Anreiz geboten, Algorithmen und Annahmen offenzulegen, so dass diese von Dritten überprüft und etwaige Fehlannahmen und Schwächen aufgedeckt werden könnten. ${ }^{125}$

Aus urheberrechtlicher Perspektive ${ }^{126}$ kommt ein Schutz für KI als Computerprogramme in Betracht. ${ }^{127}$ Algorithmen zählen zwar zu den einem Computerprogramm zugrundeliegenden Ideen und Grundsätzen, die gem. $\$ 69$ a Abs. 2 S. 2 UrhG gerade nicht vom Schutz umfasst sind. ${ }^{128}$ Ein Schutz für KI kann sich jedoch aus der konkreten Art und Weise der Einbindung von Algorithmen in ein Computerprogramm, also der Aus-

123 Zech, Technizität im Patentrecht - Eine intra- und interdisziplinäre Analyse des Technikbegriffs, Metzger, Methodenfragen des Patentrechts, FS Bodewig, 2018, S. 137,158 .

124 Kritisch auch Götting, Gewerblicher Rechtsschutz, S. 126 f. Rn. 6.

125 M. Hildebrandt, Preregistration of machine learning research design. Against Phacking, in: Being Profiled: Cogitas Ergo Sum. 10 Years of Profiling the European Citizen, 2018, S. 102, 104, abrufbar unter: https://docs.wixstatic.com/ugd/ 99e4c5_63618b010fc64c8d9055e35513802ca4.pdf, schlägt daher eine Vor-Registrierung (preregistration) von machine learning-Programmen vor.

126 Siehe hierzu Ehinger/Stiemerling, CR 2018, 761; Hartmann/Prinz, WRP 2018, 1431; Hetmank/Lauber-Rönsberg, GRUR 2018, 574, 575; Linke, Urheberrechtlicher Schutz von „KI“ als Computerprogramme - Squeezing today's innovations into yesterday's system?, in: Hetmank/Rechenberg, Kommunikation, Kreation und Innovation - Recht im Umbruch?, 2019, S. 29; Papastefanou, CR 2019, 210, 212 f.; Zech/Lauber-Rönsberg/Hetmank, GRUR Newsletter 02/2017, 17.

127 Daneben wäre noch an einen Schutz durch das sui generis Recht des Datenbankherstellers zu denken, das aber daran scheitert, dass die einzelnen Bestandteile die Algorithmen und mathematischen Modelle - keine „unabhängigen Elemente" sind. Vgl. Hauck/Cevc, ZGE 11 (2019), 135, $161 \mathrm{ff.}$

128 Vgl. BGHZ 94, 276, 285; Dreier/Schulze/Dreier, UrhG \$69a Rn. 22; Fromm/ Nordemann/Czychowski, UrhG $\$ 69$ a Rn. 30; Schricker/Loewenheim/Loewenheim, UrhG $\$$ 69a Rn. 12; Wandtke/Bullinger/Grützmacher, UrhG $\$ 69$ a Rn. 28; Hauck/Cevc, ZGE 11 (2019), 135, 160; Scheja, CR 2018, 485, 487; Söbbing, CR 2020, 223, $226 \mathrm{f}$. 
drucksform des Programms ergeben. ${ }^{129}$ Dieser Schutz erstreckt sich allerdings auch dann nicht auf die - aus technischer Perspektive entscheidenden - Algorithmen und mathematischen Modelle, sondern eben lediglich auf ihre Zuordnung zueinander. Hinzu tritt die Schwierigkeit, dass die Systeme in der Lage sind „zu lernen“, sich also selbsttätig zu verändern. Ihre genaue Funktion ist daher nicht eindeutig vorgegeben, sondern lediglich bestimmbar. ${ }^{130}$

Die Schwächen des Schutzes von Computerprogrammen spitzen sich durch den Bedeutungsgewinn von Systemen künstlicher Intelligenz zu. Nicht nur, dass die Beschränkung auf die konkrete Ausdrucksweise des Computerprogramms am eigentlichen Wert des Schutzgegenstands vorbeigeht - auch die Implikationen des Schöpferprinzips passen nicht zu der Interessenlage und dem Entstehungsprozess von Computerprogrammen. Bei der kostenintensiven Entwicklung höchstkomplexer Systeme Künstlicher Intelligenz wird dies noch einmal besonders deutlich: Hier wirkt kein Einzelschöpfer, sondern eine Vielzahl von (angestellten) Personen zusammen. Schutz wird letztlich nicht für eine kreative Leistung, sondern für die großen Investitionen begehrt, derer die Entwicklung bedarf. Gleichzeitig erfordern die Weiterentwicklung und Verbesserung von Systemen Künstlicher Intelligenz, auf bereits existierenden Systemen aufzubauen. Wie bei Computerprogrammen generell ${ }^{131}$ besteht also im Sinne der Innovationsförderung ein besonders großes Freihaltebedürfnis. Sollte demnächst über einen adäquaten immaterialgüterrechtlichen Schutz für Systeme Künstlicher Intelligenz, etwa im Rahmen eines neuen Leistungsschutzrechts, diskutiert werden, könnte dies Anlass sein, auch das (urheberrechtliche) Schutzregime für Computerprogramme zu überprüfen.

129 Vgl. Hauck/Cevc, ZGE 11 (2019), 135, 161; Linke, Urheberrechtlicher Schutz von „KI“ als Computerprogramme - Squeezing today's innovations into yesterday's system?, in: Hetmank/Rechenberg, Kommunikation, Kreation und Innovation Recht im Umbruch?, 2019, S. 29, 41. Für Computerprogramme BGH, GRUR 1991, 449, 453.

130 Vgl. hierzu Papastefanou, „Machine Learning“ im Patentrecht - Herausforderungen beim Erfinderbegriff und der Patentierfähigkeit von Algorithmen, in: Hetmank/Rechenberg, Kommunikation, Kreation und Innovation - Recht im Umbruch?, 2019, S.9, 19 f.; Linke, Urheberrechtlicher Schutz von „KI“ als Computerprogramme - Squeezing today's innovations into yesterday's system?, in: Hetmank/Rechenberg, Kommunikation, Kreation und Innovation - Recht im Umbruch?, 2019, S. 29, 43 f.

131 Vgl. Dreier/Leistner, GRUR-Beilage 2014, 13, $14 \mathrm{f}$. 


\section{Von KI generierte Gegenstände}

Auch für die Gegenstände, die eine KI generiert (ohne dass sich in dem Ergebnis noch ein gestalterischer menschlicher Wille verwirklicht), ${ }^{132}$ stellt sich die Frage, ob bzw. inwieweit sie durch das Recht des geistigen Eigentums geschützt sind. Beispiele für solche autonom geschaffenen Erzeugnisse gibt es mittlerweile viele, ${ }^{133}$ wie etwa das Gemälde Portrait of Edmond de Belamy, das durch einen Algorithmus des Kollektivs Obvious geschaffen wurde und im Oktober 2018 bei einer Auktion von Christie's für 432.500 US-Dollar versteigert wurde. ${ }^{134}$ Oder die Warnleuchte und der Lebensmittelcontainer, die von der KI „DABUS“ entworfen und beim EPA, beim UKIPO und beim USPTO zum Patent angemeldet wurden. ${ }^{135}$ Beide Beispiele erfüllen zwar in Bezug auf den Gegenstand grundsätzlich die Anforderungen an ein Werk bzw. eine Erfindung. Sie wurden jedoch nicht durch eine natürliche Person geschaffen; es fehlt an einem Schöpfer bzw. einem Erfinder. Die Patentanmeldung von Warnleuchte und Lebensmittelcontainer scheiterten deshalb in allen Ämtern daran, dass keine (natürliche) Person als Erfinder benannt werden konnte. ${ }^{136}$ Perspektivisch stellt sich aber die Frage, ob bzw. unter welchen Voraussetzungen Erzeugnisse Künstlicher Intelligenz immaterialgüterrechtlich geschützt werden sollten. ${ }^{137}$

132 Vgl. hierzu Dornis, GRUR 2019, 1252, 1253.

133 Für weitere Beispiele siehe Dornis, GRUR 2019, 1252, 1253; Yanisky-Ravid, Generating Rembrandt: Artificial Intelligence, Copyright, and Accountability in the 3 A Era - The Human-Like Authors are Already Here - A New Model, Mich. St. L. Rev. 659, 668 (2017).

134 Christie's, "Is artificial intelligence set to become art's next medium?", abrufbar unter: www.christies.com/features/A-collaboration-between-two-artists-one-human-one-a-machine-9332-1.aspx.

135 Patentanmeldungen beim EPA EP 18275163 vom 17.10.2018, EP 18275174 vom 7.11.2018; Patentanmeldungen beim UKIPO Nrn. GB1816909.4 vom 17.10.2018 und GB1818161.0 vom 7.11.2018; Patentanmeldungen beim USPTO Nr. 16/524,350 vom 29.7.2019. Vgl. Hierzu Chen, "Can an AI be an inventor? Not yet.”, MIT Technology Review, 8.1.2020, abrufbar unter: www.technologyreview.com/2020/01/08/102298/ai-inventor-patent-dabus-intellectual-propertyuk-european-patent-office-law/.

136 Siehe EPA-Entscheidung vom 27. Januar 2020 zu EP 18275 163, S. 4 ff.; EPAEntscheidung vom 27. Januar 2020 zu EP 18275 174, S. 4 ff.; UKIPO, Decision of 4.12.2019, BL O/741/19, S. 4 ff.; USPTO, Decision on petition in re application of application no.: 16/524,350, S. $4 \mathrm{ff}$.

137 Vgl. hierzu Dornis, GRUR 2019, 1252; Hetmank/Lauber-Rönsberg, GRUR 2018, 574; Legner, ZUM 2019, 807; Nägerl/Neuburger/Steinbach, GRUR 2019, 336. Auch 
Das theoretische Fundament des geistigen Eigentums - vor allem die persönlichkeitsrechtliche Begründung des Urheberrechts in Kontinentaleuropa aber auch die Anreiztheorie des Patentrechts - vermag eine solche Ausdehnung auf Werke, hinter denen keine natürliche Person mehr steht, allerdings kaum zu tragen. ${ }^{138}$ Aus diesem Grund wurde eine Regelung, wie sie der UK Copyright, Designs and Patents Act 1988 für computer-generated works vorsieht, ${ }^{139}$ schon 1990 von der WIPO als „nicht von dem Begriff des Werkes der Literatur und Kunst gemäß der Berner Übereinkunft erfaßt" abgelehnt. ${ }^{140}$

Aus Sicht der Produzenten von Systemen Künstlicher Intelligenz ist ein immaterialgüterrechtlicher Schutz für die generierten Gegenstände offensichtlich wünschenswert. Auch wird befürchtet, der durch den (oben angesprochenen) Schutz für die Systeme als solche geschaffene Investitionsanreiz laufe leer, wenn die vom System hervorgebrachten Ergebnisse schutzlos sind. ${ }^{141}$ Eine Gleichstellung „persönlicher" Schöpfungen und Erfindungen mit maschinellen erscheint aber aus verschiedenen Gründen bedenklich. Zum einen fällt die theoretische Begründung eines solchen Rechts äußerst schwer. Dies gilt nicht nur für Persönlichkeits- und Anreiztheorie, die unmittelbar an menschliche Attribute gebunden sind. Auch rechtsöko-

beim EPA stellt man sich bereits die Frage „Können KI und ML in Zukunft selbst ,erfinden', oder handelt es sich beim Erfinder per Definition um einen Menschen, der ihm zur Verfügung stehendes Werkzeug benutzt?", Volkmer/ Theißing/Owens, Entwicklungen im Bereich der Künstlichen Intelligenz und des Maschinellen Lernens aus Sicht des EPA, GRUR Newsletter 02/2017, S. 3, 6. Yanisky-Ravid/Liu, When Artificial Intelligence Systems Produce Inventions: The 3A Era and an Alternative Model for Patent Law, 39 Cardozo L. Rev. 2215 (2018), halten das traditionelle Patentrecht für KI-generierte Erfindungen hingegen für „irrelevant and inapplicable“.

138 So auch Dornis, GRUR 2019, 1252, 1258; Hetmank/Lauber-Rönsberg, GRUR 2018, 574, 576 f.; Lauber-Rönsberg, GRUR 2019, 244, 251 f.; Legner, ZUM 2019, 807, 809; Yanisky-Ravid, Generating Rembrandt: Artificial Intelligence, Copyright, and Accountability in the 3A Era - The Human-Like Authors are Already Here A New Model, 2017 Mich. St. L. Rev. 659, 706 (2017); Yanisky-Ravid/Liu, When Artificial Intelligence Systems Produce Inventions: The 3A Era and an Alternative Model for Patent Law, 39 Cardozo L. Rev. 2215, 2243 f., 2245 f. (2018).

139 UK Copyright, Designs and Patents Act 1988, Sec. 9 III: "In the case of a literary, dramatic, musical or artistic work which is computer-generated, the author shall be taken to be the person by whom the arrangements necessary for the creation of the work are undertaken."

140 WIPO, Draft Model Law on Copyright, 30 März 1990, Ziffer 127, in 1990 Industrial Property and Copyright, 241, 259.

141 Vgl. Hetmank/Lauber-Rönsberg, GRUR 2018, 574, 579 f.; Legner, ZUM 2019, 807, 812 . 
nomische Theorien des geistigen Eigentums könnten an ihre Grenzen stoßen, denn sie basieren auf der Annahme, dass die Entstehung von Immaterialgütern einer Investition bedarf, die häufig nicht getätigt würde, wenn das Immaterialgut anschließend keinen rechtlichen Schutz genösse. ${ }^{142}$ Nun bedürfen Systeme künstlicher Intelligenz zwar in der Regel einer großen Anfangsinvestition. Sie können dann aber eine potentiell unbegrenzte Anzahl von Ergebnissen produzieren, so dass die Grenzkosten für ein einzelnes autonom geschaffenes „Werk“ oder eine „Erfindung“ gen Null tendieren. Folglich lassen sich diese Gegenstände zwar noch kausal auf die Anfangsinvestition zurückführen, aber ab dem Zeitpunkt, in dem die Investition amortisiert ist, bedarf es für die weitere Produktion eigentlich keines immaterialgüterrechtlichen Schutzversprechens mehr. ${ }^{143}$ Jedenfalls ein - im Hinblick auf Schutzdauer und Schutzumfang - vollumfänglicher immaterialgüterrechtlicher Schutz scheint daher nicht angemessen. ${ }^{144}$

Auch der verfassungsrechtliche Rahmen des geistigen Eigentums spricht gegen eine Ausdehnung auf autonom geschaffene Gegenstände. Vor allem Urheber können sich nicht nur auf die Eigentumsfreiheit (Art. 14 Abs. 1 GG) berufen, sondern auch auf das Recht auf freie Entfaltung der Persönlichkeit (Art. 1 Abs. 1 i.V.m. Art. 2 Abs. 1 GG), die Kunst- und Berufsfreiheit (Art. 5 Abs. 3 und Art. 12 Abs. 1 GG). ${ }^{145}$ Daraus lässt sich ableiten, dass den Gesetzgeber auch die Pflicht trifft, einen gesetzlichen Rahmen zu schaffen, der Urhebern und Erfindern noch Raum für ihre kreative und erfinderische Tätigkeit lässt. Dieser Freiraum darf durch die exklusiven Rech-

142 Vgl. Boldrin/Levine, Review of Economic Research on Copyright Issues, 2 (2005), 45, 46 f.; Landes/Posner, The Economic Structure of Intellectual Property Law, 2003, S. 11 ff., 20; Lemley, The Economics of Improvement in Intellectual Property Law, 75 Tex. L. Rev. 989, 993 ff. (1997).

143 Vgl. auch Yanisky-Ravid, Generating Rembrandt: Artificial Intelligence, Copyright, and Accountability in the 3A Era - The Human-Like Authors are Already Here - A New Model, 2017 Mich. St. L. Rev. 659, 702 (2017). Kritisch Dornis, GRUR 2019, 1252, $1258 \mathrm{f}$.

144 Ähnlich Dornis, GRUR 2019, 1252, 1259 ff., der einen immaterialgüterrechtlichen Schutz für von KI generierte Erzeugnisse aber prinzipiell befürwortet. Vgl. auch Legner, ZUM 2019, 807, $809 \mathrm{f}$,, die sich für ein in Schutzumfang und Schutzdauer beschränktes „Nutzerrecht“ ausspricht.

145 Vgl. Dahm, Der Schutz des Urhebers durch die Kunstfreiheit, 2012, S. 140 ff.; P. Kirchhof, Der verfassungsrechtliche Gehalt des geistigen Eigentums, in: Fürst et al., FS Zeidler, 1987, Bd. 2, S. 1639, 1653 f.; Dreier/Schulze/Dreier, UrhG Einl. Rn. 39; Schack, UrhR, Rn. 90, 96; Schwartmann/Hentsch, ZUM 2012, 759, 761. 
te Anderer nicht unangemessen eingeschränkt werden. ${ }^{146}$ Genau das droht aber, wenn autonom geschaffene Gegenstände (vollen) Urheberrechtsbzw. Patentschutz beanspruchen könnten. ${ }^{147}$ Denn Algorithmen produzieren wesentlich schneller als Menschen. Dies zeigen etwa Projekte wie All Prior Art ${ }^{148}$ oder All the Music: ${ }^{149}$ Mithilfe von Algorithmen werden alle denkbaren Patentansprüche (All Prior Art) bzw. Melodien (All the Music) abgearbeitet. Ziel dieser Projekte ist es allerdings nicht, zu allmächtigen Patent- bzw. Urheberrechtsinhabern zu avancieren, sondern - im Gegenteil - die Schwächen des Systems aufzuzeigen und Erfindern und Urhebern Freiräume zu sichern. Vor dem Hintergrund, dass - insbesondere in der (Pop-)Musik - die Möglichkeiten denkbarer Kombinationen physikalisch begrenzt sind, wollen die Initiatoren möglichst viel Material einer Monopolisierung entziehen. Im Fall von All Prior Art soll dies dadurch geschehen, dass die veröffentlichten Kombinationen zu einer Vorbekanntheit und damit zum Scheitern etwaiger neuer Patente führen. All the Music wiederum speichert sämtliche mathematisch denkbaren Tonkombinationen digital und stellt sie unter Creative-Commons-Lizenzen zum freien Abruf ins Internet.

Auch wenn die von All the Music beabsichtigte Wirkung - zumindest nach geltendem deutschen Recht ${ }^{150}$ - daran scheitert, dass die produzierten Melodien nicht Ergebnis eines menschlichen Schöpfungsprozesses sind, legen die Projekte doch den Finger in eine offene Wunde des Immaterialgüterrechts: Die Befürchtung, dass es Kreativität und Innovation

146 Vgl. Auch Lemley, IP in a World Without Scarcity, 90 NYU L. Rev. 460, 510 (2015): „IP law needs to protect inventors, not just by offering them exclusive rights, but by shielding them from exclusive rights claimed by others.”

147 Vgl. hierzu Dornis, GRUR 2019, 1252, $1259 \mathrm{f}$.

148 https://allpriorart.com/. "All Prior Art is a project attempting to algorithmically create and publicly publish all possible new prior art, thereby making the published concepts not patent-able. The concept is to democratize ideas, provide an impetus for change in the patent system, and to preempt patent trolls.”

149 http://allthemusic.info/. "We've created an application to generate by brute force all mathematically possible melodies and write them to MIDI files. The application accepts various parameters (e.g., pitch, rhythm, length) to mathematically exhaust all melodies that have ever been — and are mathematically possible."

150 Zur Einordnung im US-amerikanischen Copyright Law siehe Boyden, Emergent Works, 39 Colum. J. L. \& Arts 377 (2016); Ramalho, Will Robots Rule the (Artistic) World? A Proposed Model for the Legal Status of Creations by Artificial Intelligence Systems, 21 J. Internet L. 11 (2017); Yanisky-Ravid, Generating Rembrandt: Artificial Intelligence, Copyright, and Accountability in the 3A Era The Human-Like Authors are Already Here - A New Model, 2017 Mich. St. L. Rev. 659 (2017). 
mehr behindert als fördert, weil Erfinder und Urheber übermäßig von potentiellen Verletzungsklagen bedroht sind. Die Diskussion um einen etwaigen immaterialgüterrechtlichen Schutz autonom generierter Gegenstände könnte Gelegenheit bieten, sich diesem Problem zu stellen und die geltenden Regelungen daraufhin zu überprüfen, ob sie Kreativität und Erfindungsgeist tatsächlich noch angemessen fördern.

\section{Filtertechnologien}

Technisch eng verwandt mit dem Voranschreiten von KI-Systemen sind Filtertechnologien, also Programme zur Bild-, Audio- oder Text-Erkennung. Auch hier kommen Algorithmen zum Einsatz, die mithilfe umfangreicher Datensätze erlernen, Inhalte zu „erkennen“. Sie können dann eingesetzt werden, um bestimmtes Material, etwa ein Bild oder ein Musikstück im Internet, zu identifizieren. Obwohl solche Programme bereits einen hohen Grad an Genauigkeit aufweisen und etwa in der Lage sind, Bilder, Audio-Dateien und Videos auch dann zu erkennen, wenn sie leicht modifiziert sind, sind Fehlentscheidungen - sowohl False Positives als auch False Negatives - kaum zu vermeiden. ${ }^{151}$ Zudem mangelt es den Systemen an Kontextverständnis. ${ }^{152}$ Sie können etwa die emotionale Botschaft eines Bildes oder den Humor einer Parodie nicht verstehen. Dies wird sich in absehbarer Zeit voraussichtlich auch nicht ändern. ${ }^{153}$

Diese Defizite der Technik werden dann zu einem Problem des Rechts, wenn automatisierte Entscheidungen an die Stelle menschlicher Einschätzung treten. Das ist etwa der Fall, wenn Internetplattformen Filtertechnologien einsetzen, um einer immaterialgüterrechtlichen Haftung zu entgehen. Bereits nach geltendem Recht können Host-Provider als Störer für Immaterialgüterrechtsverletzungen haften, wenn sie zumutbare Prüfungs-

151 Vgl. EuGH, Urt. v. 16.2.2012 - C-360/10 (Sabam/Netlog), CR 2012, 265 Rz. 50; Katyal/Schultz, The unending search for the optimal infringement filter, 112 Colum. L. Rev. Sidebar 83, 103 (2012); Kastl, GRUR 2016, 671, 673; Raue/Steinbach, ZUM 2020, 355, 363.

152 Vgl. Fink, ZGE 9 (2017), 288, 297 f.; Daskal, Speech Across Borders, 105 Va. L. Rev. 1605, 1625 (2019); Fremuth/Friedrich, EuZW 2019, 942, 945; Paal, JZ 2020, 91, 93 f.; Schröder, K\&R 2019, 722, 723.

153 Vgl. Daskal, Speech Across Borders, 105 Va. L. Rev. 1605, 1624 (2019); Fremuth/ Friedrich, EuZW 2019, 942, 945; Kettemann/Tiedeke, Welche Regeln, welches Recht?: Glawischnig-Piesczek und die Gefahren nationaler Jurisdiktionskonflikte im Internet, VerfBlog, 10.10.2019, abrufbar unter: https://verfassungsblog.de/wel che-regeln-welches-recht/; Paal, JZ 2020, 91, 93; Spindler, NJW 2019, 3274, 3275. 
pflichten verletzen. ${ }^{154}$ Diese Prüfungspflichten können auch erfordern, Filtertechnologien einzusetzen. ${ }^{155}$ Die Richtlinie über das Urheberrecht und die verwandten Schutzrechte im digitalen Binnenmarkt (DSM-RL) ${ }^{156}$ sieht nun für „Diensteanbieter für das Teilen von Online-Inhalten“ sogar eine Eigenhaftung vor (Art. 17). ${ }^{157} \mathrm{Um}$ einer solchen zu entgehen, müssen die Plattformen u.a. ,alle Anstrengungen unternommen [haben], um sicherzustellen, dass bestimmte Werke und sonstige Schutzgegenstände, zu denen die Rechteinhaber den Anbietern dieser Dienste einschlägige und notwendige Informationen bereitgestellt haben, nicht verfügbar sind" (Art. 17 Abs. 4 lit. b DSM-RL). Diese Voraussetzung macht, in Anbetracht der großen Mengen von Materialien, die auf den betroffenen Plattformen hochgeladen und geteilt werden, den Einsatz von Filtertechnologien fast unumgänglich. ${ }^{158}$

Die Regelung des Art. 17 DSM-RL stand massiv in öffentlicher Kritik ${ }^{159}$ und wirft in der Tat eine Vielzahl von Fragen auf. ${ }^{160}$ Hier sollen lediglich

154 BGH, GRUR 2002, 618, 619 - Meißner Dekor; BGH, GRUR 2008, 702 Rn. 51 Internetversteigerung III; BGH, GRUR 2010, 633, 634 f. - Störerhaftung des WLANInhabers; BGH, GRUR 2011, 152 Rn. 145 - Kinderhochstüble im Internet; BGH, GRUR 2011, 1038 Rn. 26 - Stiftparfüm; BGH, GRUR 2013, 370 Rn. 28 - Alone in the Dark; BGH, GRUR 2013, 1229 Rn. 36 - Kinderhochstühle im Internet II; BGH, GRUR 2013, 1030 Rn. 46 - Rapidshare.

155 Kastl, GRUR 2016, 671, 674.

156 Richtlinie (EU) 2019/790.

157 Vgl. Dreier, GRUR 2019, 771, 775; Gielen/Tiessen, EuZW 2019, 639, 643 ff.; F. Hofmann, GRUR 2019, 1219; Peifer, GRUR-Prax 2019, 403, 404 f.; Pravemann, GRUR 2019, 783, 784; G. Wagner, GRUR 2020, 447, 448 ff.; Wandtke/Hauck, ZUM 2019, 627, $632 \mathrm{ff}$.

158 Vgl. Dreier, GRUR 2019, 771, 776; Klass, ZUM 2020, 353; Kreutzer, Wie Artikel 17 der EU-Urheberrechtsrichtlinie die Let's Play-und Walkthrough-Kultur bedroht, iRights.info, 23.08.2019, abrufbar unter: https://irights.info/?p=29650; Pravemann, GRUR 2019, 783, 786; Raue/Steinbach, ZUM 2020, 355, 356; Spindler, CR 2019, 277, 285; F. Hofmann, ZUM 2019, 617, 621.

159 Vgl. Der Tagesspiegel, „Europaweiter Protest gegen neues Urheberrecht“, 23.3.2019, abrufbar unter: www.tagesspiegel.de/politik/artikel-13-europaweiter-p rotest-gegen-neues-urheberrecht/24136870.html; Zeit Online, „Sie sind keine Bots“, 23.3.2019, abrufbar unter: www.zeit.de/digital/internet/2019-03/eu-urhebe rrecht-artikel-13-demonstration-berlin-uploadfilter-youtube-save-your-internet; taz, „160.000 Bots auf deutschen Straßen“, 24.3.2019, abrufbar unter: https://taz. de/Protest-gegen-Uploadfilter/!5582669/. Nach Verabschiedung der umstrittenen Regelung reichte Polen am 24.5.2019 Klage gegen Art. 17 Abs. 4 Buchst. b und c beim EuGH ein, da diese gegen das in Art. 11 der Charta der Grundrechte der Europäischen Union verankerte Recht auf freie Meinungsäußerung und Informationsfreiheit verstießen (Rs. C-401/19, Verfahren anhängig. Vgl. Verfahrensmitteilung des EuGH, Abl. 2019, L 130, S. 92). 
zwei Aspekte von Filtertechnologien herausgegriffen werden, die mittelbar zu fundamentalen Veränderungen im Urheberrecht führen könnten: Zum einen die Schwierigkeit, den Wertungen der urheberrechtlichen Schranken auch bei Einsatz automatisierter Systeme weiterhin zur Geltung zu verhelfen, zum anderen das Erfordernis, den Systemen Material zum Abgleich zur Verfügung zu stellen.

Die Schrankenregelungen sehen zumeist nicht nur quantitative Grenzen vor, wie den Umfang der Nutzung, sondern vor allem qualitative Merkmale, insbesondere den Zweck und Kontext der Nutzung. Diese Merkmale sind für automatisierte Systeme in der Regel nicht erkennbar und würden eine menschliche Einzelfallprüfung erfordern. ${ }^{161}$ Dementsprechend besteht die Gefahr eines Overblocking, sprich dass auch urheberrechtlich zulässiges Material gesperrt wird. Dieses Problem ist allerdings nicht neu, ${ }^{162}$ sondern ein strukturelles Problem der Intermediärshaftung, ${ }^{163}$ das durch den flächendeckenden Einsatz automatisierter Systeme nur verschärft wird. Interessant ist in diesem Zusammenhang aber ein gegenläufiger Trend, nämlich die Anerkennung von Rechten der PlattformNutzer. ${ }^{164}$ Zwar normiert die DSM-RL für diese keine Ansprüche. ${ }^{165}$ Art. 17 Abs. 7 DSM-RL erkennt aber an, dass Filter-Maßnahmen nicht dazu führen dürfen, „dass von Nutzern hochgeladene Schutzgegenstände, bei denen kein Verstoß gegen das Urheberrecht oder verwandte Schutzrechte vorliegt, nicht verfügbar sind, und zwar auch dann, wenn die Nutzung ei-

160 Siehe hierzu etwa Becker, ZUM 2019, 636; Dreier, GRUR 2019, 771, 775 ff.; Gerpott, MMR 2019, 420; Pravemann, GRUR 2019, 783; Senftleben, ZUM 2019, 369.

161 Vgl. Dreier, GRUR 2019, 771, 777; F. Hofmann, GRUR 2019, 1219, 1221; Kastl, GRUR 2016, 671, 675; Raue/Steinbach, ZUM 2020, 355, 356; Schnabel, MMR 2008, 281, 283; Spindler, GRUR 2011, 101, 108.

162 Vgl. nur F. Hofmann, ZUM 2019, 617. So setzt etwa Youtube seit 2007 sein sog. Content-ID Verfahren ein, um (vermeintlich) urheberrechtsverletzendes Material zu identifizieren und zu sperren, vgl. Google, Verwendung von Content ID (abrufbar unter: https://support.google.com/youtube/answer/3244015?hl=de); G. Wagner, GRUR 2020, 329, 333.

163 Vgl. Kuschel, Netzsperren im Privatrecht, in: Mittwoch et al., Netzwerke im Privatrecht, 2016, S. 261, 288 f.; Raue, JZ 2018, 961, 963 („klassische Principal-AgentKonstellation); Raue/Steinbach, ZUM 2020, 355, 356. Siehe dazu auch oben Teil B.III.

164 Siehe hierzu in Bezug auf Meinungsbeiträge auf Internet-Plattformen Eifert, Das Netzwerkdurchsetzungsgesetz und Plattformregulierung, in: Eifert/Gostomzyk, Netzwerkrecht, 2018, S. 9, 37 f.; Jobst, NJW 2020, 11, 14; Peukert, Stellungnahme zum Netzwerkdurchsetzungsgesetz u.a. vom 12.5.2019, S. 10 ff.; Raue, JZ 2018, 961.

165 Vgl. Volkmann, CR 2019, 376, 382. 
nes Werkes oder sonstigen Schutzgegenstandes im Rahmen einer Ausnahme oder Beschränkung erlaubt ist." Für den Fall des Overblocking hat der Diensteanbieter nach Art. 17 Abs. 9 DSM-RL wirksame und zügige Beschwerde- und Rechtsbehelfsverfahren zur Verfügung zu stellen. Diese Vorgabe bewirkt eine wichtige Veränderung: Dem Nutzer wird in Bezug auf urheberrechtliche Schranken eine rechtlich bewehrte Position ${ }^{166}$ verschafft. Aus dem rein rechtfertigenden bzw. begrenzenden Charakter der Schranken ${ }^{167}$ wird, für Nutzer, die Inhalte auf entsprechenden Plattformen hochladen, ein „subjektives Recht auf Schrankennutzung“. ${ }^{168} \mathrm{Im} \mathrm{Zu}$ ge der Neuregelung der Haftung von Plattformbetreibern könnte damit ein Schritt in Richtung eines Systems getan worden sein, in dem die Interessen der Allgemeinheit - insbesondere von Personen, die selbst auf Grundlage der Werke anderer kreativ tätig werden - konkreter abgebildet sind. In den individuellen Gegenansprüchen ${ }^{169}$ der Nutzer materialisiert sich „das diffuse Interesse der Öffentlichkeit an einem offenen kreativen Kommunikationsraum"170. Führte man diesen Gedanken fort, könnte auch eine Durchsetzung der urheberrechtlichen Schranken gegenüber vertraglicher Abbedingung und technischen Schutzmaßnahmen - über die Grenzen des $₫ 95 b$ UrhG hinaus - angezeigt sein.

Diesem Interesse der Öffentlichkeit würde auch eine weitere - dem Einsatz von Filtertechnologien nachfolgende - Veränderung dienen: Damit die Systeme rechtsverletzende Inhalte aufspüren können, müssen sie zunächst wissen, wonach sie suchen. Der Rechtsinhaber muss das geschützte Material also in irgendeiner Form dem System als Referenz, etwa in einer Datenbank, zur Verfügung stellen. Diese Voraussetzung erkennt auch

166 Vgl. zu dem Begriff Zech, Vertragliche Dispositionen über Schranken des geistigen Eigentums, in: Leible/Ohly/Zech, Wissen - Märkte - Geistiges Eigentum, 2010, S. 187, 190, mit Verweis auf Alexy, Theorie der Grundrechte, 1985, S. 208.

167 Stieper, Rechtfertigung, Rechtsnatur und Disponibilität der Schranken des Urheberrechts, 2009, S. $168 \mathrm{f}$. („tatbestandliche Begrenzungen der ausschließlichen Verwertungsrechte des Urhebers“); Ullrich, ZUM 2010, 311, 312; Zech, Vertragliche Dispositionen über Schranken des geistigen Eigentums, in: Leible/Ohly/ Zech, Wissen - Märkte - Geistiges Eigentum, 2010, S. 187, $190 \mathrm{f}$.

168 F. Hofmann, GRUR 2019, 1219, 1227. Vgl. auch ders., EuZW 2020, 397, 401 f.; Volkmann, CR 2019, 376, $382 \mathrm{f}$.

169 Vgl. auch Hilty, Sündenbock Urheberrecht?, in: Ohly/Klippel, Geistiges Eigentum und Gemeinfreiheit, 2007, S. 107, 115 ff., der „Gegenschutz"-Mechanismen fordert. Kritisch gegenüber dem Ansatz, dem subjektiven Recht des Urhebers mit subjektiven (Gegen-)Rechten der Nutzer zu begegnen, Peukert, Güterzuordnung als Rechtsprinzip?, 2008, S. 72; Wielsch, Zugangsregeln, 2008, S. 62.

170 Klass, ZUM 2020, 353, 355. 
Art. 17 DSM-RL an, der die Filterverpflichtung auf „bestimmte Werke und sonstige Schutzgegenstände“ beschränkt, „zu denen die Rechteinhaber den Anbietern dieser Dienste einschlägige und notwendige Informationen bereitgestellt haben" (Abs. 4 lit. b). Offen ist dabei noch, welches Material die Rechtsinhaber bereitstellen müssen und ob die Bereitstellung bei jeder Plattform einzeln oder in (zentralen oder dezentralen) Datenbanken erfolgt. ${ }^{171}$ Ein zentrales, transparentes Register wäre jedenfalls sowohl gegenüber einem aufwändigen Einzelmeldeverfahren bei jeder Plattform als auch gegenüber einem privat geführten Register durch marktmächtige Plattformen vorzugswürdig. Dies erkennt auch die Bundesregierung an, die in ihrer Erklärung zur DSM-RL die Europäische Union auffordert, „Konzepte [zu entwickeln], die einem de-facto-Copyright-Register in der Hand marktmächtiger Plattformen durch öffentliche, transparente Meldeverfahren entgegenwirk[en]“..$^{172}$

Auch wenn die Hinterlegung von urheberrechtlich geschütztem Material in einem solchen Register aus technischer Perspektive unmittelbar einleuchtet, lässt das Wort „Register“ den Urheberrechtler doch aufhorchen. Denn mit der persönlichkeitsrechtlich orientierten Begründung des kontinentaleuropäischen Urheberrechts ist eine Registerpflicht nicht zu vereinbaren; den urheberrechtlichen Werkschutz zeichnet hiernach gerade aus, dass er unmittelbar mit dem Schöpfungsakt entsteht und jede (nicht von einer Schranke gedeckte) Nutzungshandlung durch Dritte nur mit Zustimmung des Rechtsinhabers erlaubt. ${ }^{173}$ Allerdings scheinen sowohl die persönlichkeitsrechtliche Urheberrechtstheorie als auch der daraus folgende sehr extensive Urheberrechtsschutz nicht mehr uneingeschränkt zeitgemäß. ${ }^{174} \mathrm{Im}$ Übrigen liegen zwischen strenger Registrierungspflicht als Schutzvoraussetzung und bedingungsloser Verwirklichung des Schöpferprinzips durchaus Graustufen. So existieren bereits Register für verwaiste und vergriffene Werke, ${ }^{175}$ in die - unter gewissen Voraussetzungen - privilegierte Institutionen ein Werk eintragen lassen können und nutzen dür-

171 Raue/Steinbach, ZUM 2020, 355, 358.

172 Erklärung der Bundesrepublik Deutschland zur Richtlinie über das Urheberrecht und verwandte Schutzrechte im Digitalen Binnenmarkt, insbesondere zu Artikel 17 der Richtlinie, 15. April 2019, S. 2, abrufbar unter: https://www.bmjv. de/SharedDocs/Downloads/DE/News/PM/041519_Protokollerklaerung_Richtlin ie_Urheberrecht.pdf.

173 Zum Einfluss der theoretischen Begründung des Urheberrechts auf die Wahrnehmung von Formalitäten siehe van Gompel, Formalities in Copyright Law, 2011, S. $98 \mathrm{ff}$.

174 S.o. B. IV.

175 \61a Abs. 4 UrhG, \52 VGG. 
fen, soweit und solange der Rechtsinhaber nicht widerspricht bzw., im Fall von verwaisten Werken, nachträglich ausfindig gemacht wird. ${ }^{176}$ Die Regelungen zugunsten von verwaisten und vergriffenen Werken begegnen dem Problem der Unternutzung ${ }^{177}$ urheberrechtlich geschützter Güter, das dadurch entsteht, dass Werke selbst dann nicht ohne zuvor eingeholte Erlaubnis des Rechtsinhabers genutzt werden dürfen, wenn dieser sie selbst nicht (mehr) verwertet oder gar kein Interesse an der Geltendmachung seines Urheberrechts hat. Das Recht löst dieses Problem, indem es „[a]n die Stelle eines Opt-in, das die Suchkosten vollständig auf den Nutzer verlagert, ein Opt-Out [setzt], mit dem es die Transaktionskosten zwi-

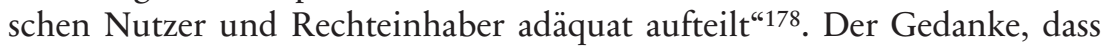
es in manchen Situationen sinnvoll und angemessen ist, die Transaktionskosten zwischen Nutzer und Rechtsinhaber aufzuteilen, indem letztere ihr Interesse an der eigenen Rechteverwertung kundtun müssen, ist keinesfalls auf den Bereich der verwaisten und vergriffenen Werke beschränkt. So hat etwa die Open-Content-Bewegung gezeigt, dass viele Urheber bereit sind, ihre Werke kostenfrei anderen zur Verfügung zu stellen. Denkbar wäre nun, diese Ausnahmen partikulär zur Regel zu machen, aus der diejenigen Rechtsinhaber, die an einer Verwertung ihrer Rechte interessiert sind, herausoptieren können. Hinzu kommt, dass neue Technologien, wie etwa die Blockchain, ${ }^{179}$ eine einfache und sichere Registrierung von Werken und die Rückverfolgung von Nutzungen und ggf. deren Monetarisierung ermöglichen. ${ }^{180}$ Das Recht könnte sich diese neuen technischen Möglichkeiten - wie schon in anderen Situationen ${ }^{181}$ - zunutze machen, um „technik-

$176 \rrbracket 61$ b S. 1 UrhG, $\$ 51$ Abs. 1 Nr. 5 VGG.

177 Vgl. hierzu Peukert, Der Schutzbereich des Urheberrechts und das Werk als öffentliches Gut. Insbesondere: Die urheberrechtliche Relevanz des privaten Werkgenusses, in: Hilty/Peukert, Interessenausgleich im Urheberrecht, 2004, S. 11, 17; Stieper, Rechtfertigung, Rechtsnatur und Disponibilität der Schranken des Urheberrechts, 2009, S. $81 \mathrm{f}$. Zum Phänomen der "Tragedy of the Anti-Commons“ siehe nur Heller, The Tragedy of the Anticommons: Property in the Transition from Marx to Markets, 111 Harv. L. Rev. 621 (1998).

178 Grünberger, ZUM 2020, 46, 51.

179 Als Blockchain wird die Sammlung von Daten in einem dezentral organisierten Verzeichnis bezeichnet, die ihre Richtigkeitsgewähr daraus ableitet, dass die Daten an allen vollständigen Netzknoten gespeichert sind und nachträglich nicht verändert werden können, vgl. Blocher, AnwBl 2016, 612, 615.

180 Vgl. Rivière, Blockchain technology and IP - investigating benefits and acceptance in governments and legislations, Junior Management Science 3(1), 2018, S. 1,7 .

181 Vgl. hierzu nur Specht-Riemenschneider, Diktat der Technik, 2019. 
sensibel“182 der Allgemeinheit die Vorteile eines transparenten Schutzsystems zu verschaffen ${ }^{183}$ und gleichzeitig die Belastung der Rechtsinhaber gering zu halten.

\section{Fazit}

Die Digitalisierung hat das Recht des geistigen Eigentums unbestreitbar vor Herausforderungen gestellt, die es bislang - mal mehr, mal weniger erfolgreich gemeistert hat. In mancher Hinsicht hat sich das Recht flexibel gezeigt, so zum Beispiel beim Aufkommen neuer Schutzgegenstände und Verwertungsarten. An anderer Stelle scheinen historisch geprägte Grundsätze, wie etwa die lange Schutzdauer und die Formfreiheit des Urheberrechts, nur schwer mit der Dynamik der Digitalisierungskultur in Einklang zu bringen. Sowohl die Realität des Werkschaffens und der erfinderischen Tätigkeit als auch die der Nutzung und Verbreitung immaterieller Güter hat sich von den theoretischen Grundannahmen weit entfernt.

Die Technologien von morgen zeigen schon erste Auswirkungen auf das Recht des geistigen Eigentums. Ob sie zu einem Umbruch führen werden, lässt sich noch nicht vorhersagen - sicher ist aber, dass sie Gelegenheit bieten, bisherige Annahmen zu hinterfragen und Schieflagen im System zu korrigieren. Das Beben, das von den digitalen Technologien ausgelöst wurde und seitdem die etablierte Ordnung des Rechts am geistigen Eigentum erschüttert hat, ist noch lange nicht vorüber. Im Idealfall gelingt es uns aber, die Bewegung, die es mit sich bringt und die Energie, die es freisetzt, zu nutzen und das Recht zukunftsfähig zu gestalten. Die wichtigste und zugleich schwierigste Aufgabe wird dabei sein, die richtige Balance zwischen den Interessen der Urheber und Erfinder, der Verwerter und Investoren und der Allgemeinheit zu finden. Diese „Zukunftsfrage des Schutzes von Immaterialgüterrechten "184 stellte sich gestern und heute. Mit großer Wahrscheinlichkeit wird sie sich auch morgen stellen.

182 Zum Begriff des techniksensiblen Urheberrechts vgl. Grünberger/Podszun, ZGE 6 (2014), 269; Grünberger, ZUM 2015, 273, 276; F. Hofmann, ZGE 8 (2016), 482 ff.; Specht, GRUR 2019, 253.

$183 \mathrm{Zu}$ den Vorteilen einer Registrierungspflicht im (digitalen) Urheberrecht vgl. Dreier/Leistner, GRUR-Beilage 2014, 13, 20 f.; Gibson, Once and Future Copyright, 81 Notre Dame L. Rev. 167, 226 ff. (2005); van Gompel, Formalities in Copyright Law, 2011, S. 3 ff. und 286 f. Kritisch Schack, Weniger Urheberrecht ist mehr, in: Bullinger et al., FS Wandtke, 2013, S. 9, 17.

184 Hoffmann-Riem, Innovation und Recht, Recht und Innovation, 2016, S. 438. 